\title{
The Beltrami SAR Framework for Multi-channel Despeckling
}

\author{
Joel Amao-Oliva, Deni Torres-Román, Israel Yanez-Vargas, Andreas Reigber, Fellow, IEEE, and Marc Jäger
}

\begin{abstract}
In this paper, a new framework for iterative speckle noise reduction in polarimetric synthetic aperture radar $(\mathrm{Pol})(\mathrm{SAR})$ data is introduced. Speckle is inherent to all coherent imaging systems and affects SAR imagery in the form of strong intensity variations in pixels with similar backscattering coefficients. This makes the interpretation of SAR data in several applications a difficult task. The proposed framework includes a pre-processing step capable of dealing with noise correlation usually found in single-look data. The general filtering approach is based on the Beltrami flow for de-noising manifolds or images painted on manifolds. The principal contribution of this work is to adapt this approach to deal with covariance or coherency matrices instead of optical imagery. The evaluation presented suggest that this approach allows for good spatial and radiometric preservation compared to other state of the art methods. Experiments are performed on the basis of synthetic and real world experimental data. The validation of the proposed framework is accomplished using two refined error performance measures and the well known effective number of looks (ENL) measured. The source code of a parallel implementation of the proposed framework is released under the MPL $2.0^{1}$ alongside this paper.
\end{abstract}

Index Terms-synthetic aperture radar (SAR), polarimetry, Beltrami flow, estimation, speckle, covariance matrix, image denoising.

\section{INTRODUCTION}

$\mathbf{R}$ EMOTE sensing refers to the acquisition of information about events or objects of the earth's surface at a distance. The acquisition of this data is achieved via passive or active sensors that capture energy reflected from the surface for later analysis, processing and interpretation for different applications like climate change, oceanography, geology, resource management, etc.

In the past years, synthetic aperture radar (SAR) technology has seen a renewed research interest with the launch of several spaceborne sensors capable of offering high-resolution, all day and weather independent imagery of the earth surface. Polarimetric SAR (PolSAR) increases the number of information channels obtained from the measured scene by means of

This work was supported by the Consejo Nacional de Ciencia y Tecnología research grant 253955 and scholarship program 280936.

J. Amao-Oliva and D. Torres-Román are with the Department of Electrical Engineering, Communications Section, Centro de Investigación y de Estudios Avanzados, Instituto Politécnico Nacional, Guadalajara 45019, Mexico (email: jamao@gdl.cinvestav.mx; dtorres@gdl.cinvestav.mx).

I. Yanez-Vargas is with the the Department of Telematics Engineering, Universidad Politécnica de Juventino Rosas, Guanajuato 38253, Mexico (email: jyanez_ptc@upjr.edu.mx).

A. Reigber and M. Jäger are with the Microwaves and Radar Institute, German Aerospace Center (DLR), Wessling D-82234, Germany (e-mail: andreas.reigber@dlr.de; marc.jaeger@dlr.de).

Manuscript received ..., 2018; revised ... .., 2019.

${ }^{1}$ https://www.mozilla.org/en-US/MPL/2.0/ combining the received back scattered signals with different transmitted wave polarizations. The resulting PolSAR data has the form of a complex target vector, containing information about the scattering process in each polarization state occurring inside each resolution cell. Since only deterministic targets can be fully defined by the scattering vectors [1], the concept of distributed scatterers arises to represent the time and space variations happening inside a resolution cell, which covariance and coherency matrices obtained from their corresponding scattering vectors can represent.

Since SAR is a coherent imaging sensor, it is affected by the presence of speckle noise, a result of the complex wave and scatterers interaction for each resolution cell. Speckle results in strong variance affecting the intensity and phase of the complex SAR data, hindering its interpretation. To mitigate the effect of speckle, multi-look processing is commonly performed; either inside the SAR processor (by dividing the available bandwidth in the frequency domain and then averaging independent samples, defined as the number of looks $L$ ) or at the final SAR image, by spatially averaging neighboring covariance or coherency matrices, reducing the speckle noise variance by a factor of $1 / L$ [2]. It is worth noting that both approaches degrade the resolution by a factor of $L$ [3].

To spatially multi-look PolSAR data, the ensemble average of $L$ independent looks (assuming the processes defining the polarization channels inside the scattering vector are stationary, jointly stationary and ergodic in mean [4]) can be replaced by the spatial average of covariance or coherency matrices of neighboring pixels. The direct application of this averaging is known as the boxcar filter, which presents high computational efficiency at the cost of highly degrading the spatial resolution.

Since the preservation of spatial details and radiometric information during speckle filtering is crucial to obtain precise knowledge about the remotely sensed scenes, the main challenge during filtering is to find an approach capable of preserving spatial resolution while at the same time reducing the presence of speckle in homogeneous areas. Moreover, the filtering of speckle in multidimensional PolSAR data needs to preserve the underlying statistical properties of the cross-product terms inside the covariance matrix and avoid introducing cross-talk between polarization channels [5].

The rest of the paper is organized as follows, Section II will present a brief summary in the field of speckle filtering and introduce the main contribution of this work. Section III reviews the data statistics for single-look and multi-look PolSAR data. A review of the short-time Beltrami kernel for images painted on manifolds is described in Section IV. Section V describes the proposed framework approach which 
is then validated in Section VI using simulated and real world datasets and three different quantitative performance metrics. Finally, conclusions about this work are summarized in Section VII.

\section{StAte of the ART AND CONTRibutions}

The well known refined Lee filter was proposed as a modified version of the Lee filter in [5] by J. -S. Lee et al. and is one of the first spatially adaptive filtering approaches. The refined approach utilizes a local linear minimum mean square error (LLMMSE) estimation within edge aligned windows in order to preserve edges and finer details present in the SAR data. This approach, while computationally inexpensive, can lead to good results as long as the spatial structures are aligned with one of the eight predefined windows. Further improvements [6] on the sigma filter were developed by Lee to overcome the deficiencies of the original sigma filter. More recently [7], Lee extended the improved sigma filter [6] to be able to filter PolSAR data. The selection of similar pixels in [7] takes into consideration the scattering properties of pixels, effectively polishing the selection of homogeneous pixels by not relying only in the complex Wishart distribution of the data.

Vasile et al. [8] proposed an intensity-driven adaptiveneighborhood (IDAN) approach that comes as an extension of Lee's work in [5] that utilizes the local morphology of the data to obtain a set of stationary pixels surrounding the center pixel instead of using edge aligned windows. The mean value of the pixels within the adaptive neighborhood is utilized as the estimated value of pixel to filter. This approach only takes into account the main diagonal of the covariance or coherency matrix to determine the set of stationary pixels disregarding any phase information during the region growing process.

D'Hondt et al. [9] proposed the adaptation of the popular bilateral filter (BLF) utilized to filter noise in gray and color images [10] to PolSAR data. The BLF uses two different kernels to obtain the weights utilized during spatial averaging. The first kernel takes into account the spatial distance between pixels whereas the second radiometric kernel measures the statistical similarity between covariance matrices inside a local window. Local approaches based on segmentation [11] and mean-shift filtering [12], [13], based on moving windows to obtain a weighted average from a homogeneous area, have all been shown to successfully suppress speckle in PolSAR data [14].

The non-local means (NLM) approach [15] has also seen some success in reducing speckle for PolSAR data, presenting good preservation of spatial details as well as a reduction of speckle noise. In their implementation, Deledalle et al. [16] presented a flexible NLM framework (NL-SAR) capable of dealing the single-look complex (SLC) or multi-look complex (MLC) imagery.

In the field of image processing, Deledalle et al. proposed the MuLoG [17] scheme to filter multi-channel SAR data. The MuLoG method is capable of taking advantage of the plethora of Gaussian de-noisers available in the literature by converting them to speckle reduction algorithms.
Stemming as well from the field of image processing, the Beltrami filter or the Beltrami short-time kernel [18]-[20] has shown promising results preserving edges and spatial features while at the same time reducing the presence of Gaussian noise in manifolds or images painted on manifolds. The Beltrami flow de-noises images by convolving them with a space-dependent kernel that is obtained from the geodesic distances found inside the image manifold. In [20], Spira et al. also found the BLF [10] as an Euclidean approximation of the Beltrami flow. Due to its effective properties as a noise filter (e.g. preservation of edges and reduction of noise in homogeneous areas), it is natural to try to extend the results of the Beltrami flow but instead only applying its core characteristics as a filter to SAR imagery.

The main contribution of this work is then the further adaptation of the Beltrami flow, referred hereinafter as the Beltrami SAR framework, described as a new speckle filtering method of multidimensional SAR data (i.e. covariance or coherency matrices) that finds similar pixels to average inside a local window, in both spatial and radiometric domains, in a similar fashion to the BLF approach [9]. However, we consider geodesic distances computed over the manifold of Hermitian positive definite matrices where we take into account the pixel connectivity component. Other contributions include the proposal of two new quantitative metrics to evaluate the performance of the proposed approach. The Beltrami SAR framework is capable of dealing with several of the common problems found inside SAR filtering, which can be summarized in a few key aspects:

1) Multi-channel support: The proposed framework allows speckle filtering of PolSAR, PolInSAR, TomoSAR, etc. data. This is possible since the distance calculation inside the Beltrami SAR filter does not care about the dimensions of the input covariance matrix. However, this work focuses on the application of the Beltrami SAR framework on PolSAR data.

2) Robustness to the data statistics: To properly filter speckle, the Beltrami SAR framework relies on a homogeneous area of simulated SAR data to estimate a parameter used as the standard deviation of speckle. To accomplish this, a simulated homogeneous area is then calculated and is filtered along side the original SAR data to obtain this parameter. In order to correctly estimate the data statistics, the filter needs the number of independent $L$ looks present in the input data to simulate $L$ independent vectors. Moreover, if noise correlation is present, the framework is able to correctly detect it and apply the same noise correlation to the simulated SAR data, in order to correctly estimate the noise statistics.

3) Single-look support: Modern speckle filtering approaches [9], [16] often require the inversion of covariance matrices to obtain the similarity measures used during averaging. To obtain the required full-rank matrices needed for inversion, multi-looking of at least $L$ independent pixels is required. This is a problem in most cases, since there is no warranty the resulting covariance matrices to filter are full rank. A pre-processing step of 
the Beltrami SAR framework allows the use of singlelook complex data, which usually presents a high-degree of noise correlation. Since no sub-sampling is performed during the pre-processing step, the Beltrami framework effectively use all the available information. The goal of this pre-processing step is then to obtain a full rank covariance matrix form rank deficient data that is subsequently filtered in the main Beltrami routine to further reduce the presence of speckle.

4) Robustness to noise correlation: During the SAR image formation process, side lobes of strong echoes that are product of unwanted reflections are reduced by the use of spectral apodization functions, this, however, spatially correlates speckle noise in the process. A popular approach to reduce or remove this spatial noise correlation is the sub-sampling of the data, at the cost of resolution. If correlation is present, the Beltrami SAR framework can take it into account.

5) Reproducible approach: To promote research and reproducibility, the Beltrami SAR framework source code has been released online under a public license. The proposed framework was implemented inside the PyRAT environment [21], and can be obtained as part of the included SAR filters.

\section{SAR DATA STATISTICS}

In a PolSAR system, the scattering vector $\mathrm{k}$ characterises the radar backscatter for a given resolution cell. This vector can be defined a (in the case of a mono-static system and using a lexicographic basis) as

$$
\mathbf{k}=\left[\begin{array}{lll}
S_{h h} & \sqrt{2} S_{x y} & S_{v v}
\end{array}\right]^{T}
$$

where $S_{h h}$ denotes the horizontally emitted and horizontally received complex scattering coefficient, $S_{v v}$ is the vertically emitted and vertically received complex scattering coefficient, $S_{x y}$ is the complex cross-polar $\left(S_{h v}=S_{v h}\right)$ polarization scattering coefficient and the superscript ${ }^{T}$ denotes the matrix transpose. In the case of SLC SAR imagery and, assuming the case of fully developed speckle within a homogeneous region [1], vector $\mathbf{k}$ follows a $Q$-dimensional [in the case of equation (1), $Q=3$ ] circular complex Gaussian distribution defined in [16], [22] as:

$$
p(\mathbf{k} \mid \mathbf{C})=\frac{1}{\pi^{Q}|\mathbf{C}|} \exp \left(-\mathbf{k}^{\dagger} \mathbf{C}^{-1} \mathbf{k}\right)
$$

where $\mathbf{C}=\mathbb{E}\left\{\mathbf{k k}^{\dagger}\right\}$ is the $Q \times Q$ complex covariance matrix associated with a pixel location, $\mathbb{E}$ represents the expected value, $|\cdot|$ is the matrix determinant and the superscript ${ }^{\dagger}$ indicates the Hermitian transpose. If the scattering vector $\mathbf{k}$ is defined in the Pauli basis instead, the resulting matrix obtained from $\mathbb{E}\left\{\mathbf{k k}^{\dagger}\right\}$ is known as the coherency matrix $\mathbf{T}[1]$.

If a lexicographic basis is used, the scattering coefficients of $\mathrm{k}$ represent single-channel SAR images, defined by an scalar complex value $z$ that follows a zero-mean, complex Gaussian distribution [16]. The amplitude of $z$, defined as $|z|$, follows a Rayleigh distribution and its intensity, $|z|^{2}$, can be described as having an exponential distribution [1]. Single-channel SAR data is said to present a multiplicative noise nature, having a quasi-deterministic radar cross section (RCS) contribution modulating a random process (speckle) [23]. It is also worth noting that speckle is not a random process, but an actual electromagnetic measurement at the sub-resolution cell level [24].

In the case of multidimensional SAR data (where $Q>1$ ), since the off-diagonal terms of the covariance matrix describe the correlations between polarization channels, the characterization of speckle noise becomes a more difficult task. For PolSAR data, Lopez-Martinez et al. [25], described speckle for diagonal terms of the covariance matrix as presenting a purely multiplicative nature, while the off-diagonal terms as having a combination of multiplicative and additive nature. This multiplicative and additive nature depends on the amount of correlation the two polarization channels have, which is described by its corresponding complex correlation coefficient $\rho[1]$.

As previously mentioned, SLC images are often multilooked to reduce the presence of speckle. This process replaces the ensemble average in equation (2) with the spatial averaging of $Y$ different neighboring scattering vectors $\mathbf{k}_{1}, \ldots, \mathbf{k}_{Y}$ for any given pixel location. The resulting MLC covariance matrix is represented as:

$$
\boldsymbol{\Lambda}=\frac{1}{Y} \sum_{i=1}^{Y} \mathbf{k}_{i} \mathbf{k}_{i}^{\dagger}
$$

where $Y$ is the effective or equivalent number of looks (ENL). The probability distribution of $\boldsymbol{\Lambda}$ when $Y \geq Q$ (this is, when the number of looks is greater or equal to the dimensionality of the scattering vector $\mathbf{k}$ ) is the so-called Wishart distribution [1], [16]

$$
\begin{aligned}
& p(\boldsymbol{\Lambda} \mid \mathbf{C})=\frac{Y^{Y Q}|\boldsymbol{\Lambda}|^{Y-Q} \exp \left(-Y \operatorname{tr}\left(\mathbf{C}^{-1} \boldsymbol{\Lambda}\right)\right)}{K(Y, Q)|\mathbf{C}|^{n}} \\
& K(Y, Q)=\pi^{(1 / 2) Q(Q-1)} \Gamma(Y) \cdots \Gamma(Y-Q+1)
\end{aligned}
$$

where $\operatorname{tr}(\cdot)$ represents the matrix trace and $K(Y, Q)$ is a normalization term. In the case of $Y<Q$, the sample covariance matrix $\mathbf{C}$ is considered to be singular (i.e. rankdeficient) and said to have a degenerate distribution [16].

\section{The Short-time Beltrami Kernel}

The main idea behind the non-linear Beltrami kernel, is the application of a space-adaptive kernel that "resides" in the signal itself, in contrast to a linear Gaussian kernel where the kernel "resides" inside the coordinate axis of the signal (see Fig. 1). This allows for a finer edge preservation and as well as a better preservation of the image details.

The Beltrami framework considers a 2-D image as an embedding $V: I \rightarrow \mathbb{R}^{N_{t}}$ with $I$ being the 2D image manifold represented by $\left\{V^{1}, V^{2}, \ldots, V^{N_{t}}\right\}=$ $\left\{u^{1}, u^{2}, \ldots, u^{J}, I^{1}, I^{2}, \ldots, I^{K}\right\}$, where $u^{i}$ are the spatial coordinates, $I^{j}$ the intensity components and $N_{t}=J+K$. $\mathbb{R}^{N_{t}}$ represents the space-feature manifold [20]. In order to filter manifolds or 2-D images defined on manifolds, Spira et 


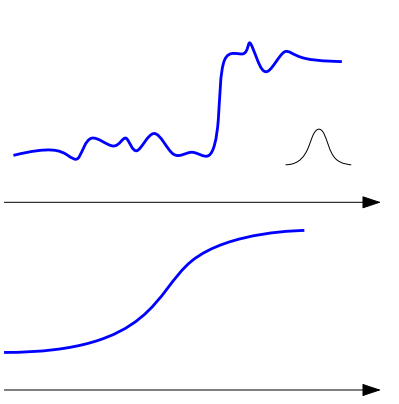

(a)
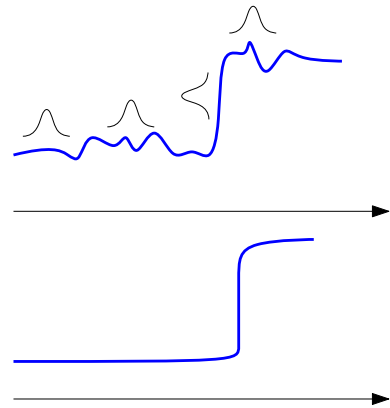

(b)
Fig. 1. Example of a 1-D signal being filtered by a Gaussian kernel in (a) versus the Beltrami kernel in (b). The Beltrami kernel can be said to reside on the signal itself while the Gaussian kernel resides in the coordinate axis of the signal.

al. [20] propose the convolution of a manifold with a spacedependent kernel for a time step size $t$ as:

$$
V^{i}\left(\mathbf{x}_{0}, t_{0}+t\right)=V^{i}\left(\mathbf{x}_{0}, t_{0}\right) *_{g} K_{B e l}\left(\mathbf{x}_{0} ; t\right)
$$

where $V^{i}$ is the space-featured image manifold and $\mathbf{x}_{0}=$ $\left(u^{1}, u^{2}\right)$ are coordinates of the center pixel. The convolution proposed in equation (5) is then defined as:

$$
V^{i}\left(\mathbf{x}_{0}\right) *{ }_{g} K_{B e l}\left(\mathbf{x}_{0} ; t\right)=\int V^{i}(\mathbf{x}) K_{B e l}\left(d_{g}\left(\mathbf{x}_{0}, \mathbf{x}\right) ; t\right) d \mathbf{x}
$$

where $\mathbf{x}=\left(\tilde{u}^{1}, \tilde{u}^{2}\right)$ are neighboring points inside the manifold and $K_{B e l}$ is the short-time Beltrami kernel [20] defined as:

$$
\begin{aligned}
K_{B e l}\left(\mathbf{x}_{0}, \mathbf{x} ; t\right) & =\frac{H_{0}}{t} \exp \left(-\frac{\left(\int_{\mathbf{x}_{0}}^{\mathbf{x}} d s\right)^{2}}{4 t}\right) \\
& =\frac{H_{0}}{t} \exp \left(-\frac{d_{g}^{2}\left(\mathbf{x}_{0}, \mathbf{x}\right)}{4 t}\right)
\end{aligned}
$$

where $\frac{H_{0}}{t}$ acts as a normalization term, $d_{g}\left(p_{1}, p_{2}\right)$ is the geodesic (i.e. the shortest path in a curved surface) distance between any two points $\left(p_{1}, p_{2}\right), d s$ defines an arc-length element on the image manifold and is equivalent to the geodesic distance $d_{g}\left(p_{1}, p_{2}\right)$.

\section{The Beltrami SAR Framework}

\section{A. The Beltrami Filter}

This section describes the adaptation of the Beltrami kernel on multi-channel SAR data in the form of $Q \times Q$ dimensional complex covariance matrices.

Instead of utilizing only radiometric and geometric information (in the case of gray or color images for the original Beltrami approach), the Beltrami SAR framework utilizes all available information of the covariance matrix. Similar to the formulation in the bilateral SAR filter [9], we can transform equation (6) into a weighted sum of covariance matrices inside a finite local window $\mathcal{W}$ centered around a pixel with coordinates defined by $\mathbf{x}_{0}$

$$
\widehat{\boldsymbol{\Lambda}}\left(\mathbf{x}_{\mathbf{0}}\right)=\sum_{\mathbf{x}_{\mathbf{i}} \in \mathcal{W}} w_{i} \mathbf{\Lambda}\left(\mathbf{x}_{\mathbf{i}}\right)
$$

where $\widehat{\Lambda}\left(\mathbf{x}_{0}\right)$ represents the estimated covariance matrix of the pixel centered at $\mathbf{x}_{\mathbf{0}}$ and $\mathbf{x}_{\mathbf{i}}$ is a neighboring pixel inside $\mathcal{W}$. The weights for each of the neighboring pixels $\mathbf{x}_{\mathbf{i}}$ are defined as:

$$
\begin{gathered}
w_{i}\left(\mathbf{x}_{\mathbf{i}}\right)=\frac{K_{S A R}\left(\boldsymbol{\Lambda}\left(\mathbf{x}_{\mathbf{0}}\right), \boldsymbol{\Lambda}\left(\mathbf{x}_{\mathbf{i}}\right)\right)}{\sum_{\mathbf{x}_{\mathbf{i}} \in \mathcal{W}} K_{S A R}\left(\boldsymbol{\Lambda}\left(\mathbf{x}_{\mathbf{0}}\right), \boldsymbol{\Lambda}\left(\mathbf{x}_{\mathbf{i}}\right)\right)} \\
K_{S A R}=\exp \left(-\frac{d_{B e l}\left(\boldsymbol{\Lambda}\left(\mathbf{x}_{\mathbf{0}}\right), \boldsymbol{\Lambda}\left(\mathbf{x}_{\mathbf{i}}\right)\right)^{2}}{\sigma^{2}}\right) .
\end{gathered}
$$

The previously defined weights have a maximum value of one if the matrices are identical and tend to zero if the matrix dissimilarity tends to infinity. In equation (10), $K_{S A R}$ represents the Beltrami SAR kernel, $\sigma$ is equivalent to the time step parameter $t$ in equation (7) $(t=1$ in our experiments) and $d_{B e l}(.,$.$) represents a new proposed distance, referred$ in this work as the Beltrami distance, that represents the integrated geodesic distance between two covariance matrices in a manifold

$$
\begin{gathered}
d_{B e l}\left(\boldsymbol{\Lambda}\left(\mathbf{x}_{\mathbf{0}}\right), \boldsymbol{\Lambda}\left(\mathbf{x}_{\mathbf{i}}\right)\right) \\
=\underset{P_{\mathbf{x}_{\mathbf{0}} \rightarrow \mathbf{x}_{\mathbf{i}}}}{\operatorname{argmin}} \sum_{(\mathbf{a}, \mathbf{b}) \in P_{\mathbf{x}_{\mathbf{0}} \rightarrow \mathbf{x}_{\mathbf{i}}}} d_{p w}\left(\boldsymbol{\Lambda}_{\mathbf{a}}, \boldsymbol{\Lambda}_{\mathbf{b}}\right) \\
P_{\mathbf{x}_{\mathbf{0}} \rightarrow \mathbf{x}_{\mathbf{i}}}=\left\{\left(\mathbf{x}_{\mathbf{0}}, \mathbf{x}_{\mathbf{1}}\right),\left(\mathbf{x}_{\mathbf{1}}, \mathbf{x}_{\mathbf{2}}\right), \ldots,\left(\mathbf{x}_{\mathbf{n}}, \mathbf{x}_{\mathbf{i}}\right)\right\} .
\end{gathered}
$$

Even though $d_{B e l}(.,$.$) is similar in application to another$ distance metrics [9] (e.g. the widely utilized Wishart distance), it differs in which it does not measure the direct distance or similarity between pair of covariance matrices, but instead finds the minimal distance path between all the pixels that exists between the two points, making $d_{B e l}(.,$.$) similar to$ the geodesic distance utilized in the original Beltrami kernel. Nevertheless, $d_{B e l}(.,$.$) still accurately measures the similarity$ between two covariance matrices.

The path $P$, described in equation (12), defines all the possible routes from the pixel location $\mathbf{x}_{\mathbf{0}}$ to the neighboring pixel $\mathbf{x}_{\mathbf{i}}$. This path is formulated as all the possible combinations of pairwise pixels that exist between the pair of covariance matrices $\left(\boldsymbol{\Lambda}_{\mathbf{a}}, \boldsymbol{\Lambda}_{\mathbf{b}}\right)$. It is important to note that all the connected points (e.g. $\mathbf{x}_{1}$ with $\mathbf{x}_{2}, \mathbf{x}_{2}$ with $\mathbf{x}_{3}$, etc.) in the path must be direct neighbors, resulting in covariance matrices that are always adjacent.

Given the high computational complexity of finding all possible geodesic paths in equation (12), the shortest geodesic path calculus in $d_{B e l}(.,$.$) is implemented as a region growing$ algorithm for finding covariance matrices inside a local window $\mathcal{W}$ (fixed to a $(2 w+1) \times(2 w+1)$ size in our experiments, for a search radio $w=3$ ) that are similar to the matrix associated with the point $\mathbf{x}_{\mathbf{0}}$ and penalizing matrices that obtain a low similarity measure score along the way (see Fig. 2). Local approaches [8], [9] cannot spatially penalize dissimilar pixels (e.g. pixels that belong to other spatial components) that are close to proximity to the reference pixel, leading 


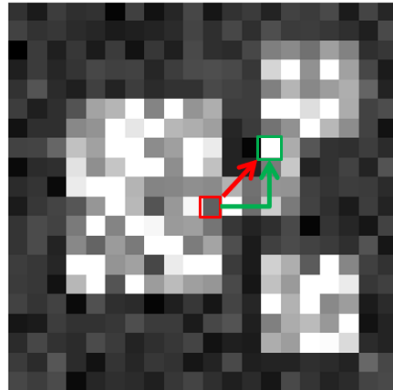

(a) Intensity image

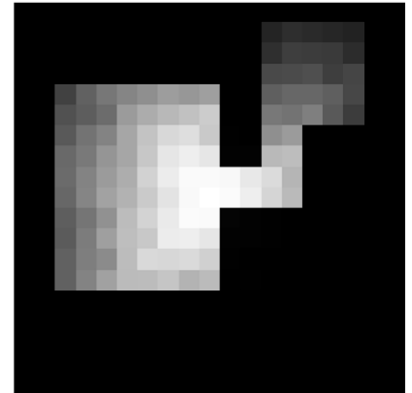

(b) Beltrami kernel

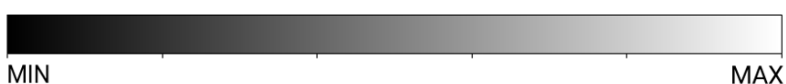

Fig. 2. Illustration showing the region growing process in the resulting Beltrami kernel (b) for several discontinuities (in the sense of sharp changes in the intensities) found in a noisy image (a). The pixel to filter belongs to the bright area in (a) marked in red. The isolated $5 \times 5$ bright patch in the lower right corner obtains an effectively zero weight value because of the sudden changes in intensities. In this example, the path in red that goes to a neighboring pixel marked in green, would result in a higher $d_{B e l}(.,$.$) distance$ compared to the path marked in green, which is the path that would end up providing the associated $d_{B e l}(.,$.$) distance for the neighboring pixel.$

to wrong estimates. The region growing implementation of the Beltrami filter takes into account spatial discontinuities, effectively discriminating spatially disconnected areas. For the region growing portion of the algorithm, only the center neighborhood window $W$ (with $w=2$ ) inside $\mathcal{W}$ is utilized to find the pairwise distances, since pixels along the edge of $\mathcal{W}$ do not require additional processing.

The pairwise distance $d_{p w}(.,$.$) , defined for a pair of adjacent$ covariance matrices $\left(\boldsymbol{\Lambda}_{\mathbf{a}}, \boldsymbol{\Lambda}_{\mathbf{b}}\right)$, is then the adaptation of the arc-length element term $d s$ found in equation (6), and is defined as:

$$
d_{p w}\left(\boldsymbol{\Lambda}_{\mathbf{a}}, \boldsymbol{\Lambda}_{\mathbf{b}}\right)=\gamma_{s}+\frac{d\left(\boldsymbol{\Lambda}_{\mathbf{a}}, \boldsymbol{\Lambda}_{\mathbf{b}}\right)}{\phi \beta}
$$

where the parameter $\gamma_{s}$ is a constant accounting for the spatial similarity between pixels. $\gamma_{s}$ is equal to 1 for neighboring pixels in any cardinal direction and equal to $\sqrt{2}$ for diagonal neighboring pixels. The parameter $\phi$ acts as a weight for the radiometric distance between two pixels and is utilized to handle the spatial correlation of noise. $\sigma$ was previously defined in equation (10). $\beta$ acts as noise standard deviation term calculated as the median of all the distances $d\left(\boldsymbol{\Lambda}_{\mathbf{a}}, \boldsymbol{\Lambda}_{\mathbf{b}}\right)$ for all pixels with another random pixel within a homogeneous region (see Section V-C1). The term $d(.,$.$) corresponds to a$ matrix distance.

\section{B. Choice of Matrix Distance}

It has been shown in previous works [9], [26] that polarimetric covariance matrices do not form a vector space but instead lie on the positive cone of a Riemannian manifold. To better deal with such properties, more fitting distance measures can be utilized. In [9], D'Hondt et al. introduced several distances suitable to obtain the weights in the BLF, some of these distances include the symmetrized Kullback-Leibler divergence [27], the affine-invariant metric [26] and a $\log$ Euclidean distance [28].

In [9], it was shown that the versions of the BLF that utilize a Riemannian metric outperform the Kullback-Leibler version of the BLF. Accordingly, this work also utilizes a Riemannian based metric to deal with the manifold structure of the covariance matrices. The Beltrami filter, however, is not fixed to any choice of matrix distance, since $d_{p w}(.,$.$) [and$ consequently $\left.d_{B e l}(.,).\right]$ can adapt to any similarity measure to obtain the shortest path distance between two points.

The chosen matrix distance that is utilized in equation (13) is then the so-called affine-invariant metric, defined as the distance between the pair of matrices $\left(\boldsymbol{\Lambda}_{\mathbf{a}}, \boldsymbol{\Lambda}_{\mathbf{b}}\right)$

$$
d\left(\boldsymbol{\Lambda}_{\mathbf{a}}, \boldsymbol{\Lambda}_{\mathbf{b}}\right)=\left\|\log \left[\boldsymbol{\Lambda}_{\mathbf{a}}{ }^{-\frac{1}{2}} \boldsymbol{\Lambda}_{\mathbf{b}} \boldsymbol{\Lambda}_{\mathbf{a}}{ }^{-\frac{1}{2}}\right]\right\|_{F}
$$

where $\log (\cdot)$ is the matrix logarithm and $\|\cdot\|_{F}$ is the Frobenius norm.

\section{Implementation Details}

1) Simulated Dataset: Since not all input data will contain homogeneous areas, and moreover, user selection of these types of zones is difficult and may not be possible for operational processing, the filter implementation uses a simulated $500 \times 500$ SAR image from $L$ complex random vectors following the approach described in [1] to allow for more freedom in the type of data to filter. As long as the simulated data is generated with the same or around the same number of looks $L$ as the input data, the measured $\beta$ parameter obtained from the simulated data will be considered as the $\beta$ value from the input data. Obtaining a correct $\beta$ estimate from the simulated dataset is important since it is directly used to filter the real dataset (i.e. the estimated noise standard deviation $\beta$ from the simulated data needs to be as close to the noise standard deviation from the real data); this also means the simulated dataset is filtered in the exact same way the real data is. The resulting formula to obtain $\beta$ is then

$\beta=\operatorname{median}\left\{d\left(\boldsymbol{\Lambda}_{i}, \boldsymbol{\Lambda}_{z}\right) \mid \boldsymbol{\Lambda}_{i}, \boldsymbol{\Lambda}_{z} \in \Omega\right\} ; i, z=0, \ldots, K_{x} \times K_{y}$

where $K_{x} \times K_{y}$ is the total number of pixels inside the simulated homogeneous region $\Omega$ and $\boldsymbol{\Lambda}_{z}$ and $\boldsymbol{\Lambda}_{i}$ are random matrices inside $\Omega$. The median is utilized over the mean to reduce the impact of outlier distances. Nonetheless, if the input data contains large enough spatial noise correlation, the simulated SAR data will fail to accurately represent the actual SAR input data.

To deal with this, the image spectrum is estimated for each available channel and then averaged together to obtain a final estimation of the spectra. This spectrum is then applied to the simulated data. After obtaining the associated image statistics, the simulated homogeneous area can accurately represent the original SAR data, from the number of looks it has, to the speckle noise correlation that could be introduced during image formation. 


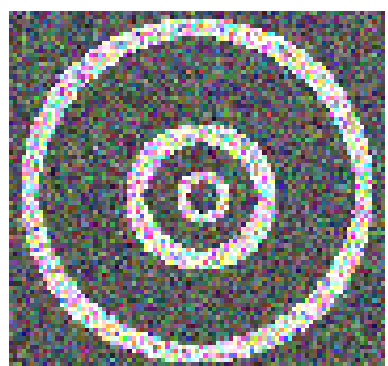

(a)

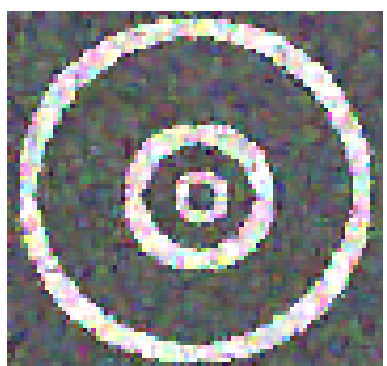

(b)

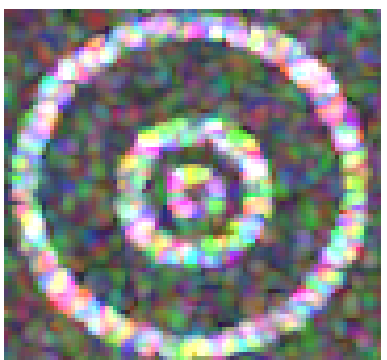

(c)

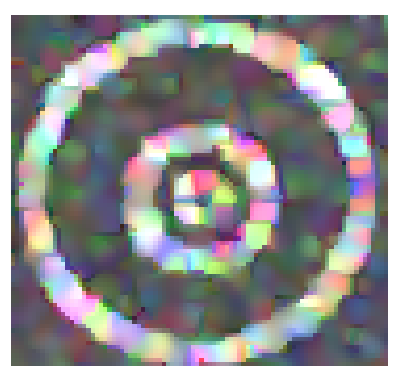

(d)

Fig. 3. Results after applying the pre-processing step on a simulated PolSAR SLC image represented with Pauli RGB color coding. (a) and (c) represent the SLC image with and without spatially correlated speckle, respectively. The filtered results in (b) and (d) represent the multi-looked (MLC) output that acts as the input to the Beltrami filter.

2) Pre-processing Step: The Beltrami SAR framework allows the use of either a SLC or MLC SAR image to obtain the corresponding filtered image. If an SLC image is used as an input, the data is then said to be rank deficient since the number of looks $Y$ is less than the dimensionality of $\mathbf{k}$. Since the distance utilized in equation (14) requires the use of full-rank matrices, a necessary pre-processing step is required to enforce the existence of said full-rank matrices. In order to obtain the needed full-rank matrices to process the SLC image, a $Q \times Q$ boxcar filter is applied to a copy of the input data. The filtered copy (i.e. the full rank distance array) of the SLC image is then utilized to obtain a first approximation of the associated Beltrami distances used in the main Beltrami filter in equation (8) using an iterative scheme (see Section $\mathrm{V}-\mathrm{C} 3$ ), until the required effective number of looks (i.e. when $Y \geq Q)$ is achieved.

3) Iterative Approach: To progressively refine the denoised estimation and consequently increase the number of looks, an iterative approach is performed as a modification of equation (8):

$$
\widehat{\boldsymbol{\Lambda}}^{[n+1]}\left(\mathbf{x}_{\mathbf{0}}\right)=\sum_{\mathbf{x}_{\mathbf{i}} \in \mathcal{W}} w_{i} \widehat{\boldsymbol{\Lambda}}^{[n]}\left(\mathbf{x}_{\mathbf{i}}\right)
$$

If the pre-processing is applied to the data, image quality is not greatly affected by the use of a boxcar filter, since the framework only utilizes the full rank distance array containing the Beltrami distances $d_{B e l}(.,$.$) instead of the whole boxcar$ filtered data array to obtain a full-rank estimate. Once the data reaches the required ENL (i.e. when the filtered matrices are full rank), the pre-processing stage stops and the Beltrami distances are calculated normally, without the use of a boxcar filter. Fig. 3 shows the results after the pre-processing step. For each iteration, $\beta$ (measuring the equivalent noise standard deviation) is updated and decreases as more iterations are performed. If the input data is in the form of an MLC image, the pre-processing step is skipped, no boxcar filter is applied and the data is assumed to have sufficient looks (i.e. it is said to be full rank). Instead of performing a rank test [9] to determine whether a pixel belongs to a point-target or not, by virtue of the region growing implementation in the filter, neighboring pixels to point-targets are given low weight values and are effectively ignored during averaging.
4) Robustness to Noise Correlation: During the filtering process, our method creates regions of similar pixels in order to obtain a de-noised estimate, similar to the non-local approach [16]. If speckle correlation is present, the data has effectively less number of independent samples compared to the number of averaged pixels. This results in the over-estimation of the similarities of neighboring pixels and the need of more samples to obtain the same smooth result compared to data without correlated noise. Given that noise correlation can easily be detected in the simulated homogeneous area, our method can account for it and adjust the $\phi$ parameter in equation (13) (in charge of controlling sensitivity of filtering) depending on the level of correlation that is detected. The empirical relation between the spatial correlation and $\phi$ is:

$$
\phi=-1.5 \Psi^{1.5}+\phi_{0}
$$

where $\phi_{0}$ is the base sensitivity parameter and $\Psi=\left(C_{A}+\right.$ $\left.C_{R}\right) / 2$ ), $C_{A}$ and $C_{R}$ are the correlation lengths of azimuth and range directions (computed from a small homogeneous area [1]), respectively, is the averaged value of the spatial correlation for adjacent pixels in the azimuth and range directions. $\Psi$ is calculated only at the first iteration of the pre-processing stage.

Equation (17) was obtained by performing a quadratic regression on a set of experimental values calculated in Section VI that resulted in the best performance of the Beltrami SAR framework in the case of $3 \times 3$ PolSAR data. The calculus of the $\phi$ parameter can be further improved by the study of higher dimensionality datasets (InSAR, TomoSAR, etc.) and the quadratic regression utilized could be exchanged by a more precise formulation, however, for the study case of this paper, equation (17) gives reasonable results.

In the case of single-look data, the Beltrami filter tends smear edges and over-filter fine details when a high level of spatial correlation is present. However, if the value of $\phi$ is modified, the filter will then increase the distance obtained in equation (13). This reduces the weight value of spatially correlated pixels, however, it comes at the cost of more iterations needed to obtain a final result, since spatial correlation results in an effectively lower number of independent averaged samples during each iteration. 


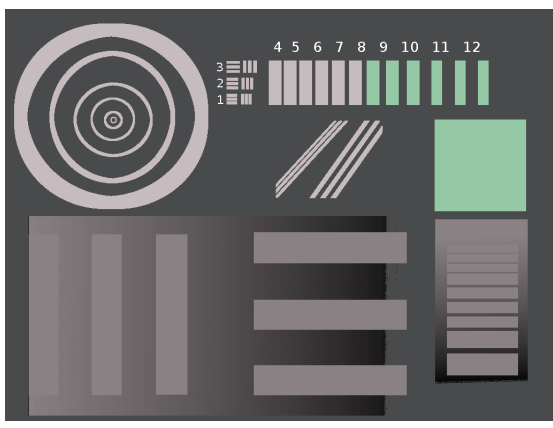

(a)

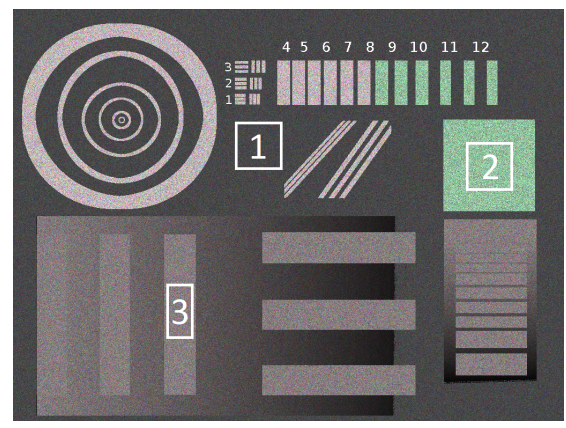

(b)

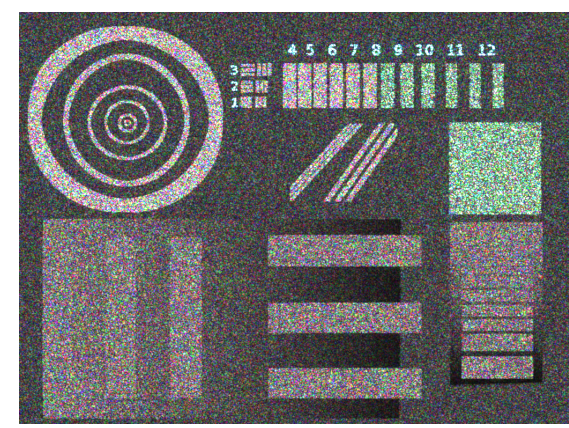

(c)

Fig. 4. $1030 \times 768$ synthetic PolSAR images used for evaluation. The ground truth is represented in (a), with several different areas and spatial features (using Pauli RGB color coding). The image in (b) represents 3-look MLC without any noise correlation while the white rectangles represent the three homogeneous areas used for evaluation in Section VI-B. Figure (c) represents the second case a single-look SAR image with an approximate spatial correlation factor $\Psi=0.5$ in both azimuth and range direction.

5) Free Parameters: The equation that fundamentally defines the Beltrami filter is the pairwise distance in equation (13). Inside the pairwise distance, the function of its main parameters $(\phi$ and $\beta)$ has been previously discussed. Since these parameters are not automatically set, further discussion on the derivation of the user-fixed values for these parameters is presented in this section.

a) $\phi_{0}$ : Inside $\phi$, this parameter controls the base spatial sensitivity used during filtering. Using the computed correlation lengths, $\Psi$ is obtained to account for the spatial correlation of the data, as discussed in the previous section. If matrix regularization is skipped (i.e. MLC input data), the value of $\phi$ will be equal to the base sensitivity $\phi_{0}$, since no spatial correlation is taken into account. Based on our experimental results (Fig. 7), a value of around 2.1 for $\phi_{0}$ results in a good balance of edge preservation and a high number of looks with a fast convergence on both synthetic and real-world results (see Section VI-A for details).

b) $L$ : In the case of multi-look data, the number of looks $(L)$ in the filter acts as a free parameter in charge of controlling the amount of spatial filtering. The initial value of $\beta$ depends on the initial simulated homogeneous area, which in turn depends on the initial value of $L$ selected in the algorithm. In Fig. 11, the first two rows show how a different initial value of $L$ results in more or less preservation of details and texture (i.e. a larger or smaller initial $L$ ), as well as a higher amount of noise smoothing.

It is worth noting, that all other quantities in the filter are either derived from these two parameters (e.g. $\phi$ from $\phi_{0}$ ) or measured directly from real or simulated data ( $\Psi$ and $\beta$ ).

6) Computational Complexity: As seen in equation (14), our approach requires the eigendecompositions, inversions and multiplications of matrices, which are shown to have an overall computational complexity on the order of $\mathcal{O}\left(Q^{3}\right)$ [29]. It is worth noting that for the Beltrami approach, the computational burden will be directly impacted by the number of iterations performed over the simulated, where the image dimensions are fixed to $M \times N=500 \times 500$, and real datasets. Thus, for a given image size of $M \times N$, the computational complexity of the Beltrami filter algorithm is in the order of $\mathcal{O}\left(M N W Q^{3}\right)$, where $W$ is the size of the local window. In case the pre- processing stage is executed, an additional factor of $Q^{2}$ needs to be applied to all complexities since the required $Q \times Q$ covariance matrices need to be estimated.

In comparison to other methods, our approach has a similar complexity to the NL-SAR and BLF approaches as well as similar running times. The addition of the region growing strategy in our method has an negligible impact, in terms of runtime, compared to the computation of the polarimetric distances which dominates the computational burden.

\section{EXPERIMENTAL RESULTS}

In order to evaluate the performance of the proposed Beltrami framework, we have performed several simulation experiments following two main experimental cases using synthetic data. For the first case, we use a simulated 3-look MLC PolSAR data generated following the fully developed speckle model and target vectors using the Gaussian distribution [1]. This image includes several edges and structures which are difficult for speckle filters to properly filter.

Effects of spatially correlated data are then explored as a second experimental case, where an SLC image is generated as before and an additional step, introduces spatial correlation using image statistics obtained from real word data, Figs. 4(b) and (c) show the first and second case, respectively.

To obtain quantitative results for the two main experimental cases, three performance metrics are presented, following [9], that describe closeness to the original data utilizing an affineinvariant metric [28], preservation of edges and amount of smoothing inside homogeneous areas. A comparison with the most prominent state of the art despeckling methods with respect to the preservation of several important radiometric parameters is presented. Finally, a qualitative analysis is performed for multiple single and multi-look real world experimental data.

\section{A. Simulation Experiments Details}

In order to determine the performance of our method, simulated datasets were needed to cover the different commonly encountered problems during SAR speckle filtering. These synthetic datasets were obtained following the same approach 


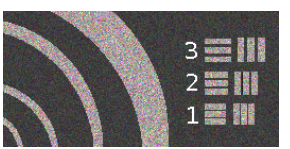

(a)

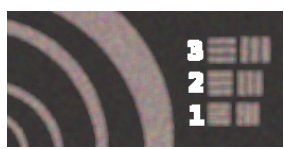

(b)

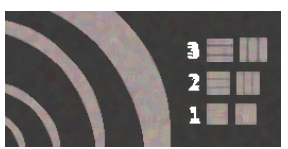

(c)

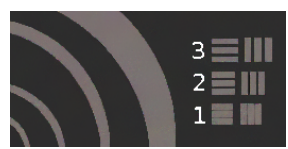

(d)

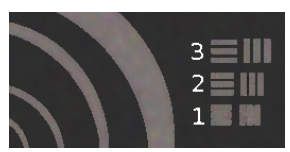

(e)

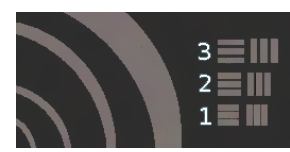

(f)

Fig. 5. Results over a cropped area comparing different speckle filtering techniques for the first synthetic case seen in Fig. 4(b). The original 3-look MLC is seen in (a). A $5 \times 5$ boxcar filter was applied in (b) resulting in the blurring of fine details in the numbers and rectangles, which are also lost using a refined Lee filter in (c). The filtered results from the NL-SAR framework and IDAN filter, in (d) and (e) respectively, show good smoothing of homogeneous areas and preservation of detail in the numbers, however some details are still lost. The result from our proposed method is showed in (f), where the filter achieves sharp edges and highly smoothed homogeneous areas.

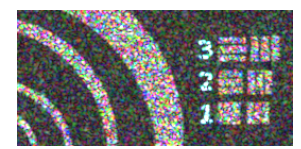

(a)

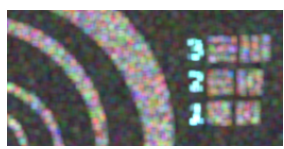

(b)

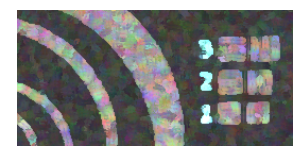

(c)

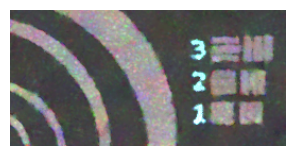

(d)

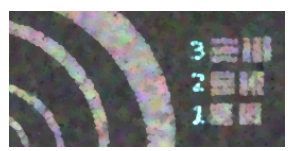

(e)

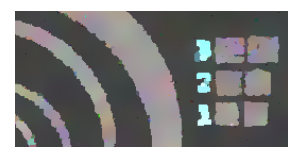

(f)

Fig. 6. Results over a cropped area comparing different speckle filtering techniques for the second synthetic case seen in Fig. 4(c). The spatially correlated SLC SAR imagery is presented in (a). In order to accommodate for the spatial correlation in the image, a $5 \times 5$ boxcar filter was applied in (b). The filtering result of the refined Lee filter is showed in (c). Again, the results from the NL-SAR framework and IDAN filter in (d) and (e) respectively, show a moderate amount of smoothing of homogeneous areas and minimal loss of details. The result from our proposed method is showed in (f), where the filter achieves good edge reconstruction, however, the pre-processing step introduces artifacts that modify the small details and edges in the final result.

as in Section V-C1. The underlying covariance matrices utilized to simulated the scattering vectors were measured from real data (point targets [shown as single bounce scattering in the numbers from 1-12], forest [volume scattering shown in green], grass and roads [single bounce scattering shown as pink and gray] are all shown in Fig. 4).

During processing, we set the step parameter $\sigma$ to 1 in order to allow consistency across all iterations. For the first synthetic case $\phi=2.1$ according to equation (17), since no spatial correlation exists in the simulated data. In the second synthetic case, $\phi=1.56$ due to spatial correlation. The framework stops iterating once the calculated noise standard deviation error $\epsilon=\left|\beta_{[n-1]}-\beta_{[n]}\right|$ (where $\beta_{[n-1]}$ and $\beta_{[n]}$ are the $\beta$ parameters calculated from the previous and current iteration, respectively) is less than a fixed tolerance. In practice, this tolerance is set to a very low value ( 0.01 in our experiments) that ensures that there is no more perceptible change to the image upon convergence.

An average of 20 iterations are needed in most cases to reach convergence, however, it depends on the type of input data and the amount of spatial correlation. It is important to note that the filter converges on a good solution based on two factors: the convergence of both global and edge errors (Fig. 7a and $7 \mathrm{~b}$ ) and the incremental effective number of looks (in Fig. 7c) across different base sensitivity values (see Section VI-B for details). Together, these results show that the data becomes increasingly smooth within homogeneous areas while, at the same time, edge contrast is preserved.

\section{B. Performance Metrics}

For an objective evaluation of the de-noising capability of the presented framework, we have adopted a quality metric that was originally [26], [28] utilized to measure the similarity between Riemannian covariance matrices which are symmetric positive-definite (i.e. tensors) in nature. The log-Euclidean metric utilized to measure similarity between covariance matrices is similar to the affine-invariance distance previously defined in equation (14) but presents a lighter computational complexity while preserving the same affine-transformation invariance. The invariance property is useful because it can be applied regardless of the type of data polarization or polarization basis utilized. Using a log-Euclidean metric to measure the global similarity between the original and filtered data results in the global similarity $(G S I M)$ measure defined as:

$$
G S I M=\frac{1}{N Q^{2}} \sum_{i=1}^{N}\left\|\log [\widehat{\boldsymbol{\Lambda}}]_{\mathbf{i}}-\log [\boldsymbol{\Lambda}]_{\mathbf{i}}\right\|_{F}
$$

where $N$ is the total number of pixels in the image, $\widehat{\Lambda}_{\mathbf{i}}$ is the estimated (i.e. filtered) covariance matrix at pixel $i$ and $\Lambda_{\mathbf{i}}$ is the associated true (speckle free) covariance matrix. A lower result represents a lower average distance (i.e. a lower reconstruction error). In order to quantify the performance of our method for edge reconstruction (and similar in construction to the proposed measure for edge reconstruction in [9]), we restrict the GSIM measure to pixels that reside in both sides of discontinuities. The resulting equation is then defined as the edge similarity $(E S I M)$ measure:

$$
\operatorname{ESIM}=\frac{1}{Q^{2}} \frac{\sum_{i=1}^{N} \delta\left(M_{i} \neq 0\right)\left\|\log [\widehat{\mathbf{\Lambda}}]_{i}-\log [\boldsymbol{\Lambda}]_{i}\right\|_{F}}{\sum_{i=1}^{N} \delta\left(M_{i} \neq 0\right)}
$$

where $M_{i}$ represents the binary mask for pixel at location $i$. The mask is set to 1 if the pixel $i$ has at least one other pixel neighboring a different class in the ground truth. $\delta()=$.1 if its argument is true. The last quality measure is the well known ENL, defined as:

$$
E N L=\frac{\widehat{\mu}^{2}}{\widehat{\sigma}_{0}^{2}}
$$




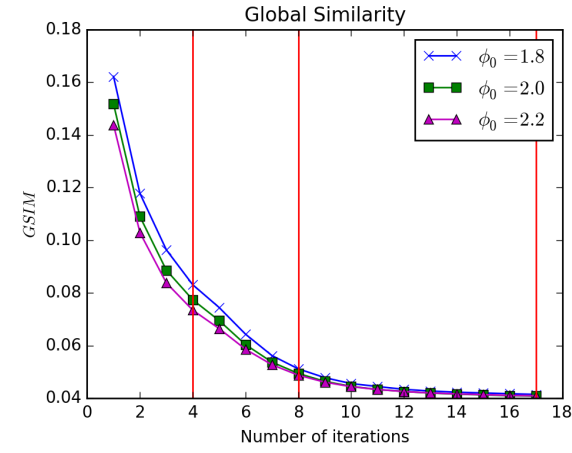

(a)

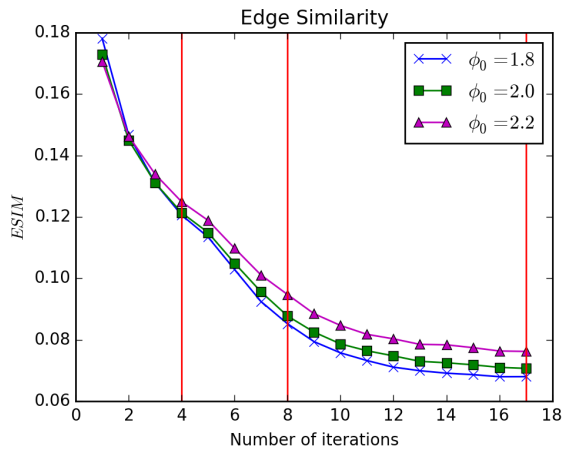

(b)

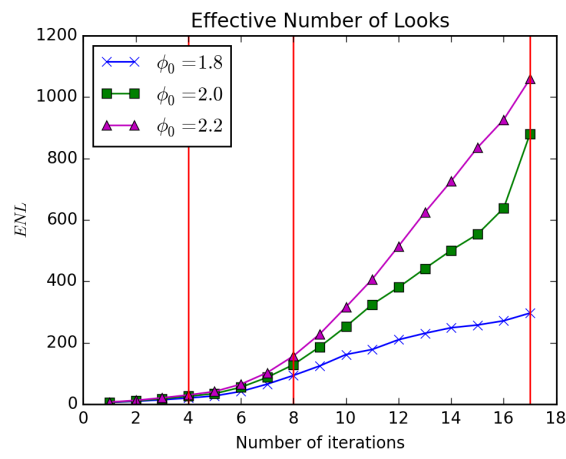

(c)

Fig. 7. Performance of the proposed framework using different base sensitivities $\phi_{0}$ over the first synthetic case. The three vertical lines in red show results at different points of filtering: after a couple of iterations $\left(N_{\text {iter }}=4\right)$, approaching convergence $\left(N_{i t e r}=8\right)$ and at convergence $\left(N_{\text {iter }}=17\right)$.

TABLE I

Comparison in Terms of Three Performance Measures for Different Speckle Filtering Technigues in Two Synthetic Cases

\begin{tabular}{c|c|c|c|c|c|c} 
& \multicolumn{2}{|c|}{$G S I M$} & \multicolumn{2}{c|}{$E S I M$} & \multicolumn{2}{c}{$E N L$} \\
\hline Method & MLC & SLC & MLC & SLC & MLC & SLC \\
\hline Boxcar & 0.069 & 0.182 & 0.24 & 0.264 & 144.9 & 5.3 \\
\hline Ref. Lee & 0.075 & 0.230 & 0.22 & 0.338 & 72.3 & 4.8 \\
\hline NL-SAR & 0.044 & 0.141 & 0.104 & 0.217 & 498.5 & 27.2 \\
\hline IDAN & 0.045 & $\mathbf{0 . 1 3 1}$ & 0.077 & $\mathbf{0 . 1 7 8}$ & 148.4 & 7.9 \\
\hline Proposed Method & $\mathbf{0 . 0 4 0}$ & 0.156 & $\mathbf{0 . 0 7 0}$ & 0.353 & $\mathbf{7 6 2 . 6}$ & $\mathbf{9 6 . 7}$
\end{tabular}

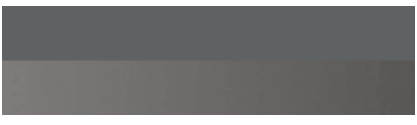

(a)

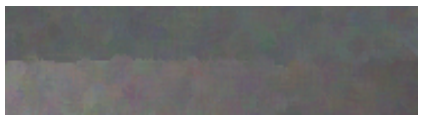

(c)

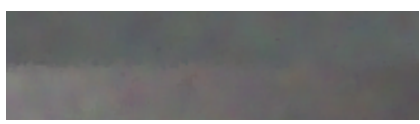

(e)

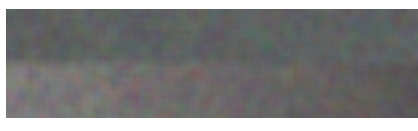

(b)

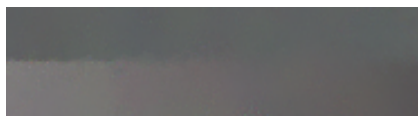

(d)

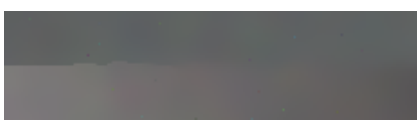

(f)
Fig. 8. Results over a cropped area for the first synthetic case. The image in (a) shows a section of the ground truth where a low contrast edge is present. Images (b)-(f) show the filtered result from the boxcar, ref. Lee, NL-SAR, IDAN and Beltrami methods, respectively. Our approach shows a comparable edge preservation to the non-local method as well as highly smoothed homogeneous areas.

where $\widehat{\mu}$ is the estimated mean and $\widehat{\sigma}_{0}^{2}$ is the estimated variance calculated over a chosen channel. Since all channels are filtered the same in our method, only the first diagonal term of $\widehat{\Lambda}$ is needed to compute the effective number of looks. The estimation of ENL allows for a measure on the amount of smoothing of homogeneous areas. To obtain the estimated ENL, homogeneous areas (1), (2) and (3) from Fig. 4b are used for evaluation and then averaged. The complex correlation parameter $\rho$ (also known as the polarimetric coherence) gives second order information about the correlation coefficients between polarimetric channels. The complex correlation parameter $\rho$ is then defined [1] as:

$$
\rho_{x y}=\frac{\mathbf{T}_{x y}}{\sqrt{\mathbf{T}_{x x} \mathbf{T}_{y y}}}
$$

where $x y$ are the available polarimetric channel pairs.

\section{Synthetic Cases Results}

For the two synthetic cases, we chose to compare the results of our method with a $5 \times 5$ boxcar filter, the refined Lee filter using $7 \times 7$ edge oriented windows and LLMMSE averaging, the NL-SAR framework with a search radius of 12 and a patch size of 5 and the IDAN filter using a neighborhood size $N_{\text {pix }}$ of 50 pixels. All the implementations of the previously mentioned methods were obtained in the PyRAT software available at [21].

Fig. 5 shows the visual comparison of the results obtained using the different de-noising methods for the first case. A cropped version of the complete filtered images is showed for visualization purposes. Table I presents the results from the first synthetic case in the columns defined as MLC for the three different quality measures. All methods demonstrate good de-noising performance according to the resulting ENL, with the proposed method on top. The result from the boxcar filter shows blurring of the edges and finer details. The limited number of aligned windows for the refined Lee method results in loss of the finer details in the numbers but a good edge preservation over edges that match one of the aligned windows. The results from the NL-SAR and IDAN methods are very similar, both visually and in the performance measures, 
TABLE II

Comparison of Mean Estimates of Eigenvalues, Entropy, Mean Alpha, Anisotropy, Diagonal Coherency Matrix elements and the Polarimetric Complex Correlation $\rho_{x y}$ OVer Three Different Homogeneous Areas for the First Experimental Case. (A) Zone 1; (B) ZONE 2; (C) ZONE 3

\begin{tabular}{c|c|c|c|c|c|c} 
& Ground Truth & Boxcar & Ref. Lee & NL-SAR & IDAN & Beltrami \\
\hline$\lambda_{1}$ & 0.17 & 0.17 & 0.16 & 0.17 & 0.16 & 0.16 \\
\hline$\lambda_{2}$ & 0.07 & 0.07 & 0.07 & 0.07 & 0.07 & 0.07 \\
\hline$\lambda_{3}$ & 0.06 & 0.05 & 0.05 & 0.06 & 0.06 & 0.06 \\
\hline$H$ & 0.9 & 0.89 & 0.89 & 0.9 & 0.9 & 0.9 \\
\hline $\bar{\alpha}$ & 0.99 & 1 & 1 & 0.99 & 1 & 0.99 \\
\hline$A$ & 0.05 & 0.14 & 0.13 & 0.07 & 0.08 & 0.06 \\
\hline$T_{11}$ & 0.09 & 0.09 & 0.09 & 0.09 & 0.08 & 0.09 \\
\hline$T_{22}$ & 0.09 & 0.09 & 0.09 & 0.09 & 0.09 & 0.09 \\
\hline$T_{33}$ & 0.11 & 0.11 & 0.11 & 0.11 & 0.11 & 0.11 \\
\hline$\rho_{12}$ & $0.31 \angle 1.90^{\circ}$ & $0.30 \angle 1.04^{\circ}$ & $0.30 \angle 1.15^{\circ}$ & $0.31 \angle 1.11^{\circ}$ & $0.30 \angle 0.82^{\circ}$ & $0.30 \angle 1.16^{\circ}$ \\
\hline$\rho_{13}$ & $0.29 \angle 22.74^{\circ}$ & $0.29 \angle 23.91^{\circ}$ & $0.29 \angle 25.85^{\circ}$ & $0.29 \angle 23.65^{\circ}$ & $0.29 \angle 23.58^{\circ}$ & $0.30 \angle 23.37^{\circ}$ \\
\hline$\rho_{23}$ & $0.36 \angle 19.72^{\circ}$ & $0.37 \angle 19.32^{\circ}$ & $0.35 \angle 21.19^{\circ}$ & $0.37 \angle 19.42^{\circ}$ & $0.35 \angle 19.06^{\circ}$ & $0.37 \angle 19.20^{\circ}$
\end{tabular}

(b)

\begin{tabular}{c|c|c|c|c|c|c|} 
& Ground Truth & Boxcar & Ref. Lee & NL-SAR & IDAN & Beltrami \\
\hline$\lambda_{1}$ & 2.96 & 3 & 2.97 & 2.99 & 2.69 & 2.92 \\
\hline$\lambda_{2}$ & 0.32 & 0.33 & 0.34 & 0.33 & 0.32 & 0.32 \\
\hline$\lambda_{3}$ & 0.25 & 0.23 & 0.24 & 0.25 & 0.25 & 0.25 \\
\hline$H$ & 0.51 & 0.5 & 0.5 & 0.5 & 0.53 & 0.51 \\
\hline $\bar{\alpha}$ & 1.12 & 1.12 & 1.12 & 1.12 & 1.11 & 1.12 \\
\hline$A$ & 0.11 & 0.17 & 0.17 & 0.12 & 0.13 & 0.11 \\
\hline$T_{11}$ & 0.69 & 0.69 & 0.69 & 0.69 & 0.65 & 0.67 \\
\hline$T_{22}$ & 0.87 & 0.87 & 0.87 & 0.88 & 0.81 & 0.86 \\
\hline$T_{33}$ & 1.97 & 2 & 1.98 & 2 & 1.81 & 1.96 \\
\hline$\rho_{12}$ & $0.66 \angle 7.34^{\circ}$ & $0.66 \angle 7.36^{\circ}$ & $0.65 \angle 7.38^{\circ}$ & $0.66 \angle 7.34^{\circ}$ & $0.64 \angle 7.28^{\circ}$ & $0.66 \angle 7.32^{\circ}$ \\
\hline$\rho_{13}$ & $0.68 \angle 5.86^{\circ}$ & $0.68 \angle 5.59^{\circ}$ & $0.68 \angle 5.56^{\circ}$ & $0.68 \angle 5.62^{\circ}$ & $0.66 \angle 5.57^{\circ}$ & $0.68 \angle 5.68^{\circ}$ \\
\hline$\rho_{23}$ & $0.75 \angle-1.42^{\circ}$ & $0.75 \angle-1.31^{\circ}$ & $0.75 \angle-1.35^{\circ}$ & $0.76 \angle-1.33^{\circ}$ & $0.74 \angle-1.25^{\circ}$ & $0.76 \angle-1.26^{\circ}$
\end{tabular}

\begin{tabular}{c|c|c|c|c|c|c} 
& Ground Truth & Boxcar & Ref. Lee & NL-SAR & IDAN & Beltrami \\
\hline$\lambda_{1}$ & 0.85 & 0.86 & 0.85 & 0.84 & 0.83 & 0.86 \\
\hline$\lambda_{2}$ & 0.4 & 0.42 & 0.42 & 0.41 & 0.4 & 0.4 \\
\hline$\lambda_{3}$ & 0.32 & 0.29 & 0.29 & 0.31 & 0.31 & 0.32 \\
\hline$H$ & 0.91 & 0.9 & 0.9 & 0.91 & 0.91 & 0.91 \\
\hline $\bar{\alpha}$ & 0.94 & 0.96 & 0.95 & 0.95 & 0.95 & 0.94 \\
\hline$A$ & 0.1 & 0.17 & 0.17 & 0.13 & 0.13 & 0.11 \\
\hline$T_{11}$ & 0.54 & 0.53 & 0.53 & 0.53 & 0.52 & 0.54 \\
\hline$T_{22}$ & 0.46 & 0.47 & 0.47 & 0.46 & 0.46 & 0.47 \\
\hline$T_{33}$ & 0.57 & 0.57 & 0.57 & 0.57 & 0.56 & 0.56 \\
\hline$\rho_{12}$ & $0.34 \angle-1.76^{\circ}$ & $0.34 \angle-1.64^{\circ}$ & $0.34 \angle-2.39^{\circ}$ & $0.34 \angle-1.42^{\circ}$ & $0.34 \angle-1.57^{\circ}$ & $0.34 \angle-1.53^{\circ}$ \\
\hline$\rho_{13}$ & $0.28 \angle 12.97^{\circ}$ & $0.27 \angle 12.74^{\circ}$ & $0.26 \angle 13.85^{\circ}$ & $0.27 \angle 13.15^{\circ}$ & $0.26 \angle 13.16^{\circ}$ & $0.29 \angle 13.04^{\circ}$ \\
\hline$\rho_{23}$ & $0.31 \angle 8.81^{\circ}$ & $0.30 \angle 8.99^{\circ}$ & $0.29 \angle 9.93^{\circ}$ & $0.30 \angle 9.04^{\circ}$ & $0.29 \angle 9.32^{\circ}$ & $0.31 \angle 8.96^{\circ}$
\end{tabular}

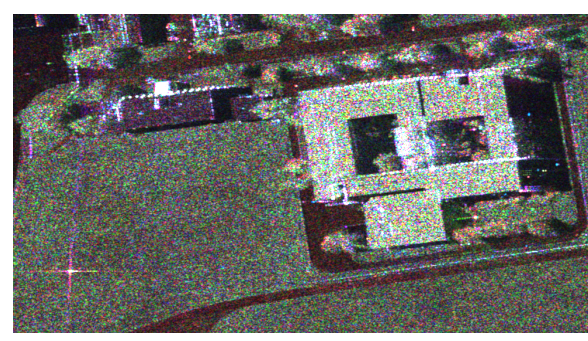

(a)

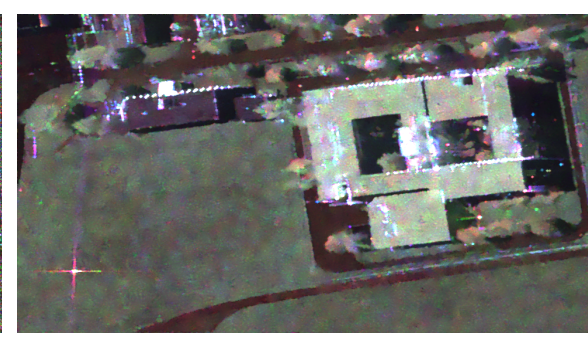

(b)

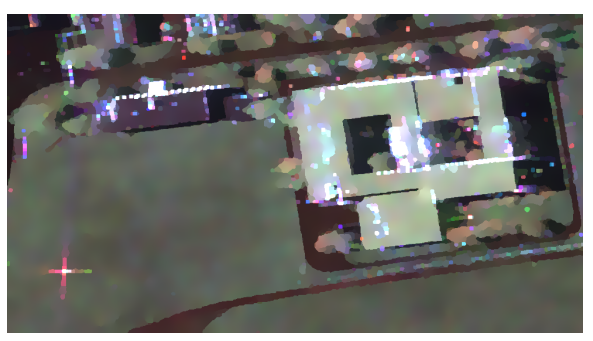

(c)

Fig. 9. SLC PolSAR image from the X-band F-SAR sensor (DLR-HR) Kaufbeuren dataset displayed using the Pauli RGB color coding. The image in (a) shows spatially correlated speckle. Results using the NL-SAR framework are shown in (b) and using the Beltrami SAR framework in (c).

with the IDAN filter outperforming the NL-SAR framework in every measure except in the ENL. The results from the Beltrami SAR framework are superior to the other methods in all the proposed performance measures.

The visual results of the second synthetic case, where the effects of spatial correlation on SLC data are explored, is presented in Fig. 6. In this experiment, due to speckle correlation and the reduction of independent number of samples, all methods show worse performance in all the presented metrics, 


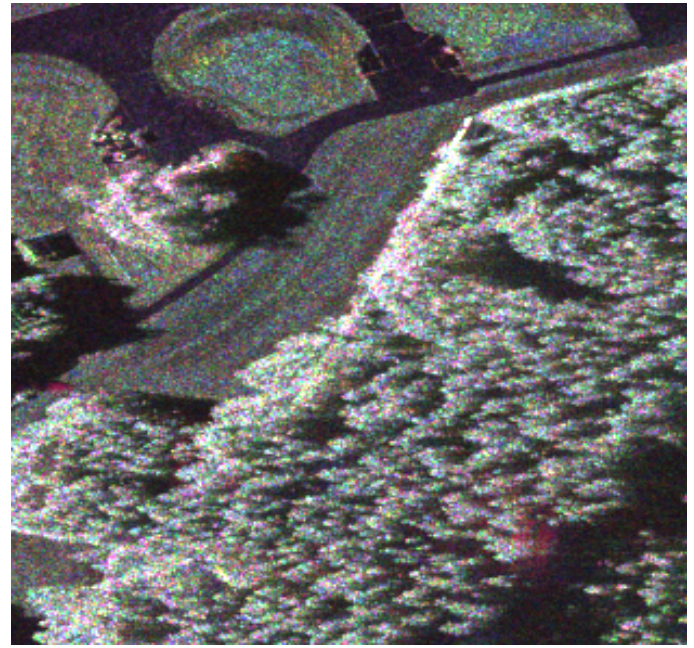

Fig. 10. Fully polarimetric X-band image of the Kaufbeuren campaign. Speckle can be clearly observed on the grass patches and over the forest.

as expected. For this experimental case, Table I presents the results in the columns defined as SLC.

The GSIM results for the NL-SAR, IDAN and the proposed method are fairly similar, showing a good overall estimation of the true covariance matrices. In the case of the ESIM results, the performance of the proposed method is poor compared to results of the IDAN and NL-SAR approaches. This could be due to the pre-processing step introducing artifacts that are carried over to the final results, lowering the performance metric result. It is worth considering that the same ground truth was utilized to calculate the ESIM in both synthetic cases. This means the edges in the SLC image are shifted to a small degree compared to the original ground truth, which is caused by the introduction of spatial correlation in the simulation experiment. Our method, however, results in a much higher smoothing over homogeneous areas, which can be explained partly due to its iterative nature.

In addition of the proposed performance metrics, we obtain the mean values for the Entropy/Mean-alpha angle/Anisotropy $(H / \bar{\alpha} / A)$ decomposition as well as the associated eigenvalues over three homogeneous areas (see Fig. 4b) and compare them to the true values using the available simulated ground truth data. This comparison allows for an analysis on the effects of speckle filtering on the polarimetric information.

Table II shows the estimated values for the proposed method and other speckle filters. Comparing the estimated values for all the methods to the original ground truth values, we see a close resemblance in the coherency matrix diagonal terms, eigenvalues, complex correlation terms and in the $H / \bar{\alpha} / A$ decomposition parameters. Since the anisotropy value $A$ is more sensitive to the presence of speckle noise, the estimated values of $A$ using the boxcar and refined Lee approaches result a slightly bigger error comparing them to the original ground truth data. The anisotropy estimation is improved using more complex spatially adaptive methods such as IDAN, NL-SAR or the proposed framework. Based on the estimation of the extracted polarimetric parameters, the Beltrami SAR framework shows no degradation of the polarimetric information and compares favorably to other state of the art approaches.

\section{Real World Data Results}

Fig. 9 shows the performance of the Beltrami SAR framework in regards to filtering SLC data where speckle correlation is present. The results from the NL-SAR framework (Fig. $9 \mathrm{~b})$ show a superior preservation of details and texture and a good reduction of speckle. The Beltrami approach (Fig. 9c) results in a much higher smoothing of homogeneous areas and an exceptional preservation of edges, nonetheless, it can be seen over-filtering some low contrast edges. As part of the evaluation process, we analysed the bright point-target near the left part of the image, finding its full preservation in both azimuth and range directions. However, the shape of its impulse response function is slightly modified due to spatial averaging during filtering.

To properly evaluate our approach, two different values for $L$ were used, as well as different intermediate results (after a certain, fixed number of iterations $N_{\text {iter }}$ ), to illustrate the possible filter outcomes. In both cases (Fig. 11c and 11f), the filter converges on a smooth piece-wise constant result. The size of the piece-wise constant areas is seen to depend on the initial spatial smoothing given by $\phi_{0}$ and $L$. Smaller piece-wise constant areas, obtained for larger values of $L$, correspond to higher spatial and textural preservation (as seen in Fig. 11f), while using a smaller $L$ leads to a much higher ENL, however, at the cost of some loss of spatial resolution (see Fig. 11c) as well as loss of textural information. Similarly, it is possible to limit the number of iterations (see Figs. 11a$11 \mathrm{~b}$ and 11d-11e) to obtain an images with improved texture preservation.

In comparison to other approaches, the Beltrami approach is flexible in that it allows the acquisition of a highly smoothed final result or results that preserve spatial resolution and textural information to different degrees, at the expense of noise reduction, while assuring convergence in all cases. Particularly, the preservation of textural information in Fig. 11f is on par with the preservation of the other approaches (Figs. 11g-11i).

The dataset used in Fig. 12 corresponds to the fully polarimetric X-band Kaufbeuren (Germany) image obtained with the F-SAR sensor in 2015 during the 15OP15AF campaign with a processed azimuth and range resolution of $25 \mathrm{~cm}$. In order to obtain a the required 4-look MLC input data, the image in Fig. 12a has been pre-summed by four pixels in both azimuth in range directions. The results from the experiment show that the filtered data (seen in Fig. 12b) obtained from the proposed Beltrami SAR framework greatly reduces the presence of speckle noise while preserving edges and point like structures. Homogeneous areas are completely smoothed out. The Beltrami filter, however, can sometimes aggressively over-smooth areas where the contrast between edges is not high enough. This can be seen in the train tracks on the upper part of Fig. 12 and in the loss of some textural detail in the forest areas. Nonetheless, this over-smoothing can be seen as a fundamental limitation of pixel-based (i.e. local) despeckling approaches [8], [9], where a trade-off between noise suppression and loss of low contrast edges (or textural information) is always present. 


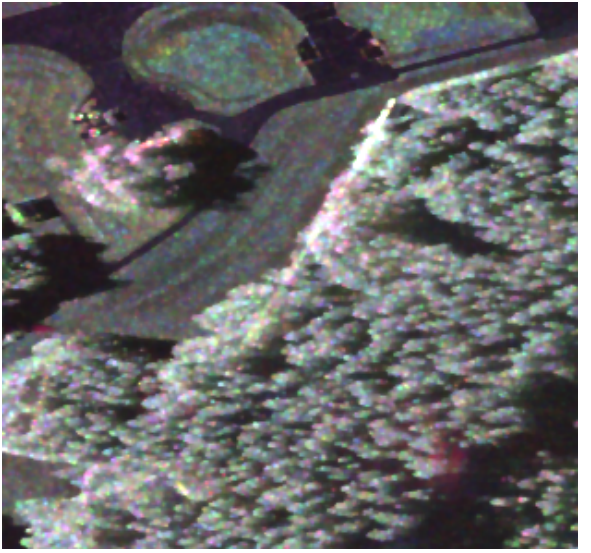

(a) $L=3, N_{\text {iter }}=4, E N L \approx 9.5$

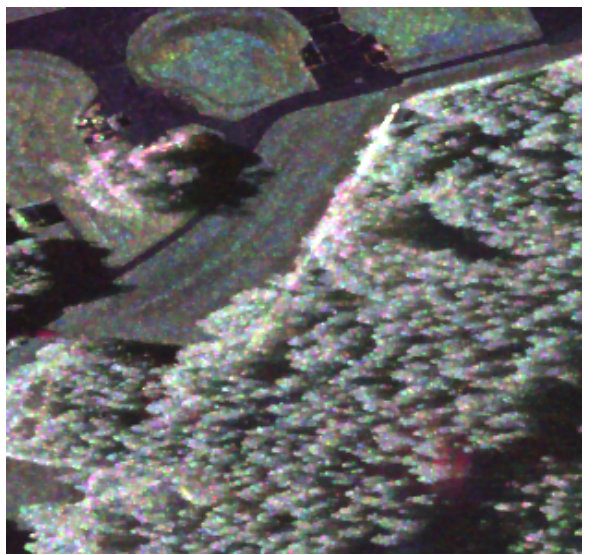

(d) $L=4, N_{\text {iter }}=4, E N L \approx 6.7$

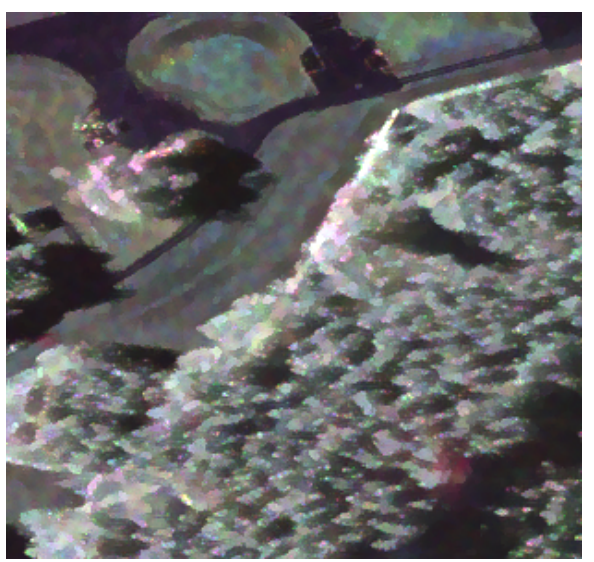

(g) Ref. Lee $(7 \times 7)$ window, $E N L \approx 22.2$

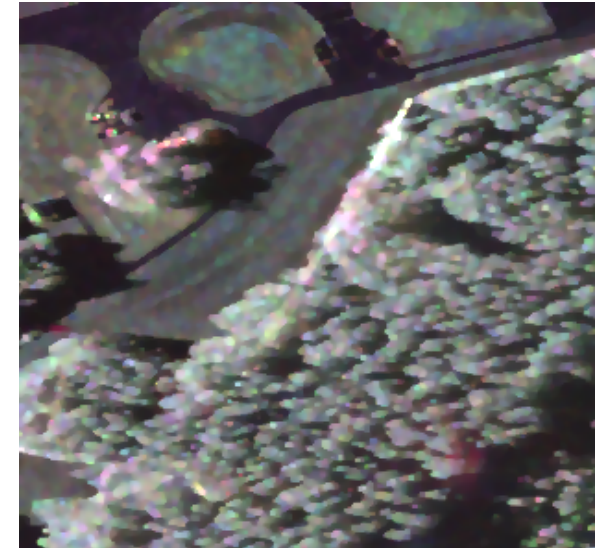

(b) $L=3, N_{\text {iter }}=8, E N L \approx 32$

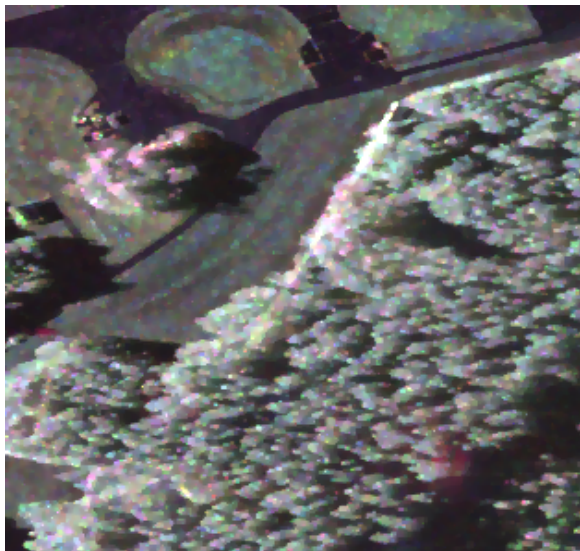

(e) $L=4, N_{\text {iter }}=8, E N L \approx 15.7$

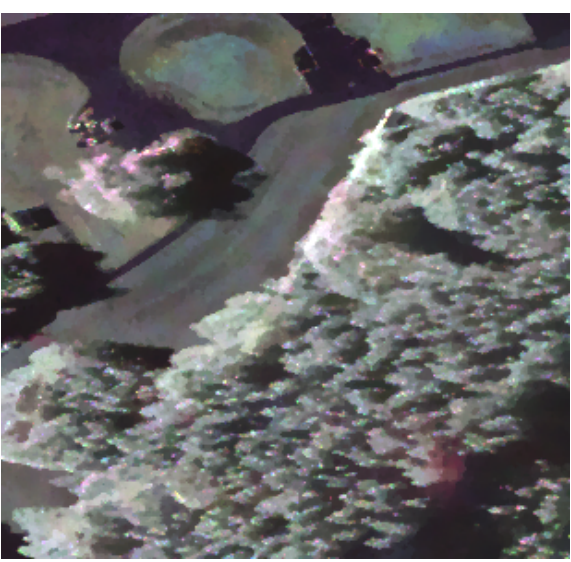

(h) IDAN, $E N L \approx 62$

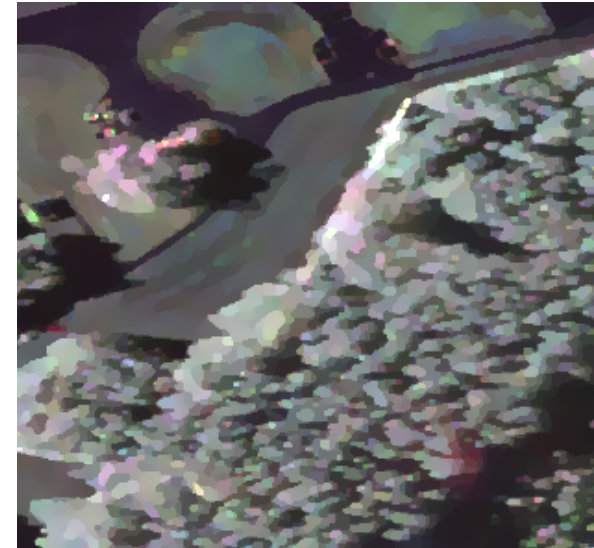

(c) $L=3, N_{\text {iter }}=17, E N L \approx 264$

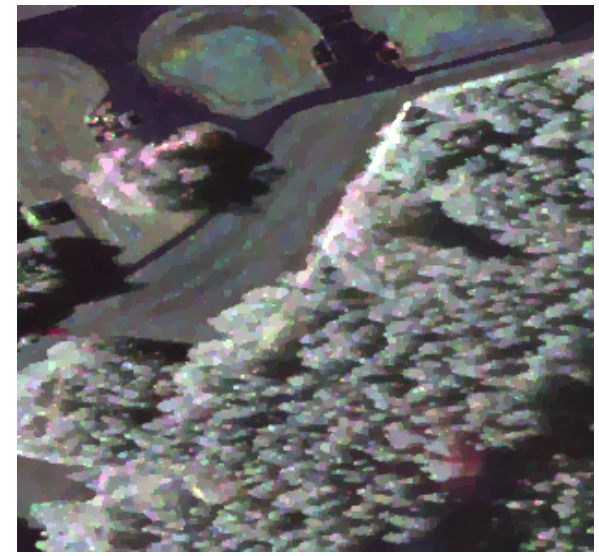

(f) $L=4, N_{\text {iter }}=17, E N L \approx 39.7$

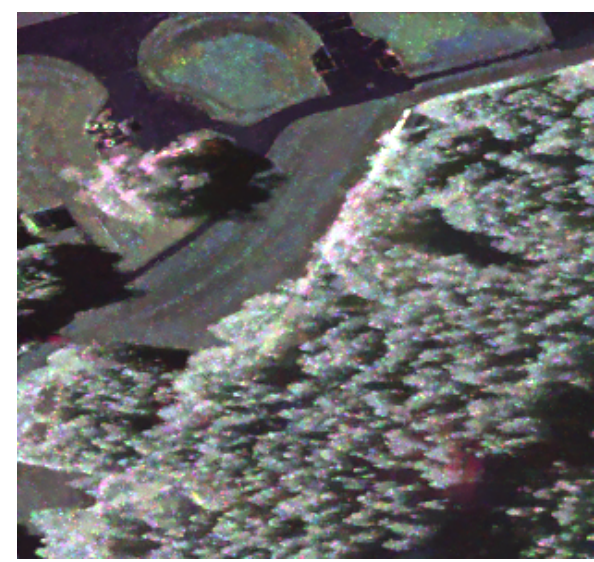

(i) NL-SAR, $E N L \approx 12.5$

Fig. 11. Results from filtering the multi-looked (via sub-sampling, see Fig. 10) Kaufbeuren image. The first two rows [from (a) to (f)] show the Beltrami framework results using two different initial number of looks $(L \in\{3,4\})$ and with the columns representing different iteration number. A base sensitivity $\phi_{0}=2.1$ was used for all experiments. For comparison, filtering results from the ref. Lee, IDAN and NL-SAR filters are shown from (g) to (i), respectively.

To visually assess the preservation of low and high contrast edges, Fig. 13 shows the results using different speckle filtering approaches in another multi-looked PolSAR dataset using the lower frequency L-band. The results from the Beltrami filter (Fig. 13f) are consistent with the other experiments, showing superior smoothing of homogeneous areas and preservation of edges. The zoomed image in the field crop reveals the main weakness of the Beltrami SAR framework, in the form of a slight textural loss and some amount of over-smoothing.

\section{CONCLUSIONS}

In this work, we developed a novel speckle filtering approach. The proposed method is based on short-time Beltrami kernel utilized in the field of image processing. The Beltrami flow presents attractive characteristics as a SAR speckle filter, particularly edge preservation and smoothing of homogeneous areas. The pre-processing step of the Beltrami framework allows the use of single-look complex data, which usually 


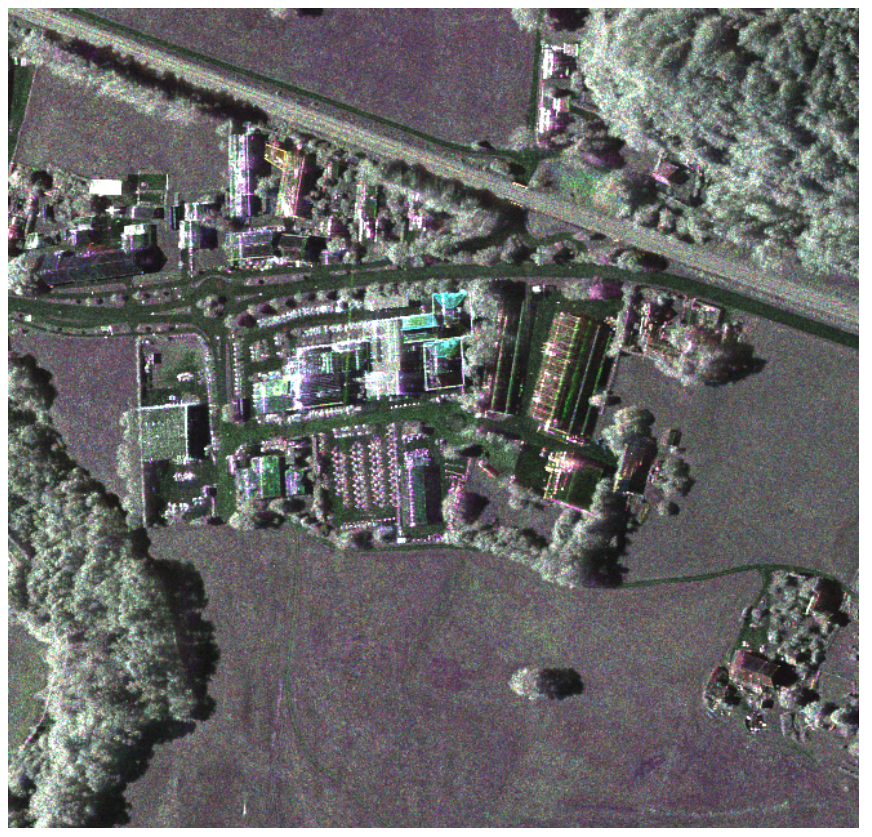

(a)

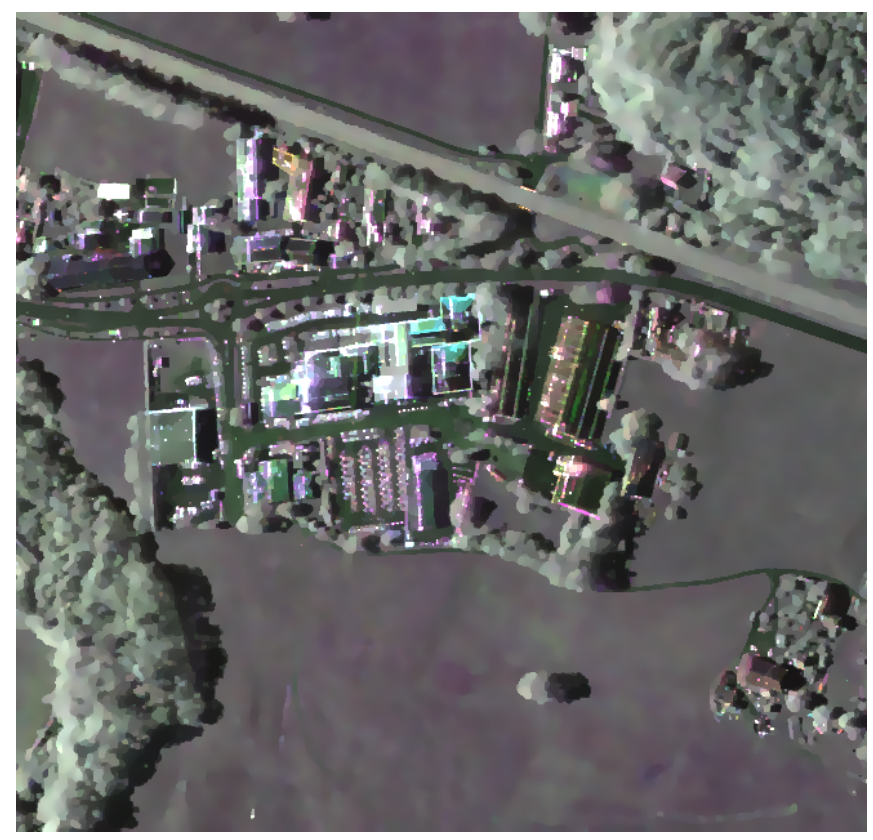

(b)

Fig. 12. Filtered polarimetric results from the X-band F-SAR (DLR), obtained from the Kaufbeuren 15OP15AF campaign, displayed using Pauli RGB color coding.

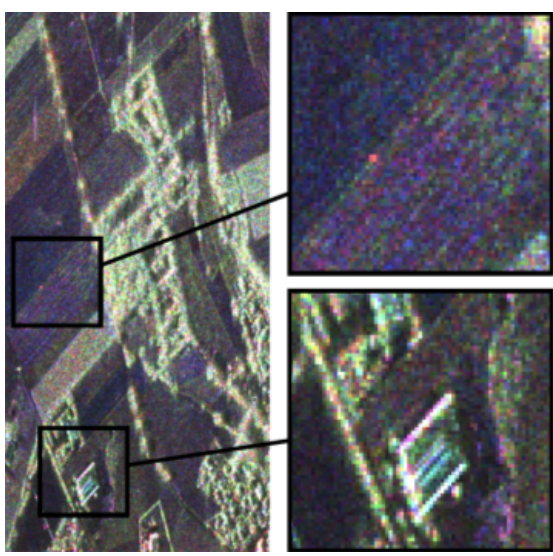

(a) Original 3-look image

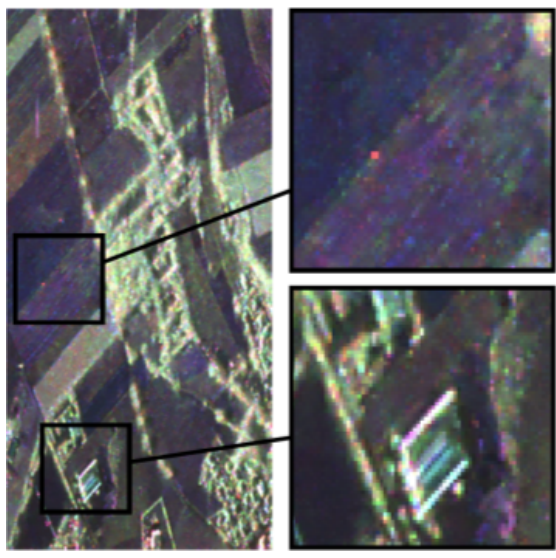

(d) NL-SAR

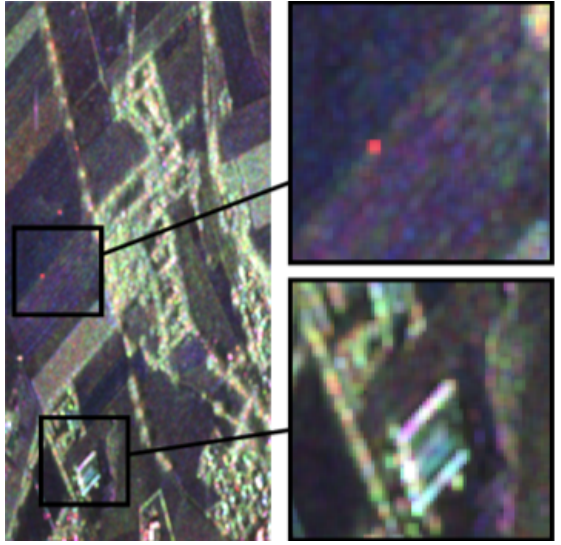

(b) $3 \times 3$ Boxcar

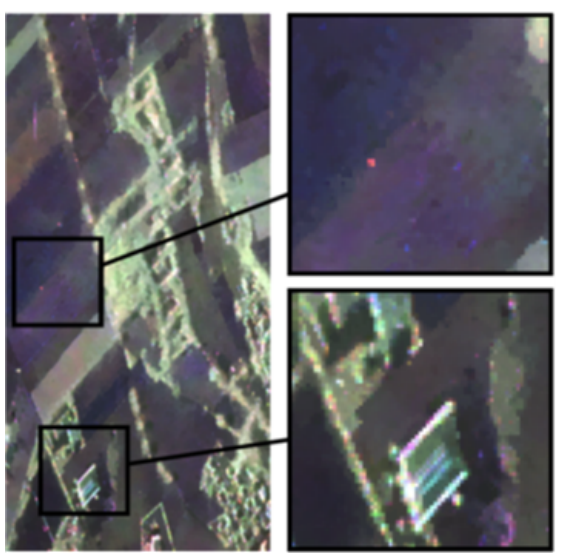

(e) IDAN

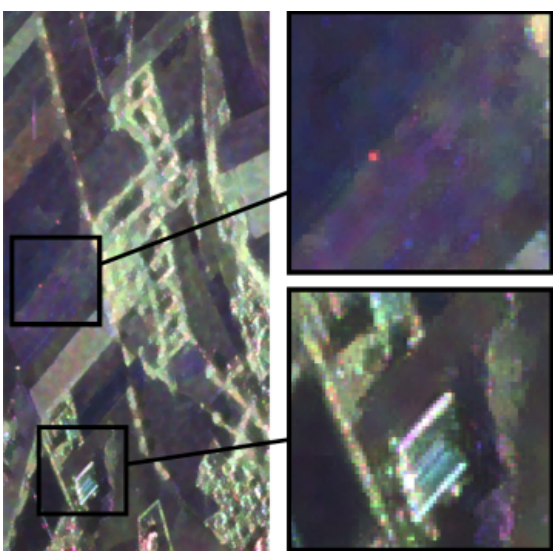

(c) Ref. Lee

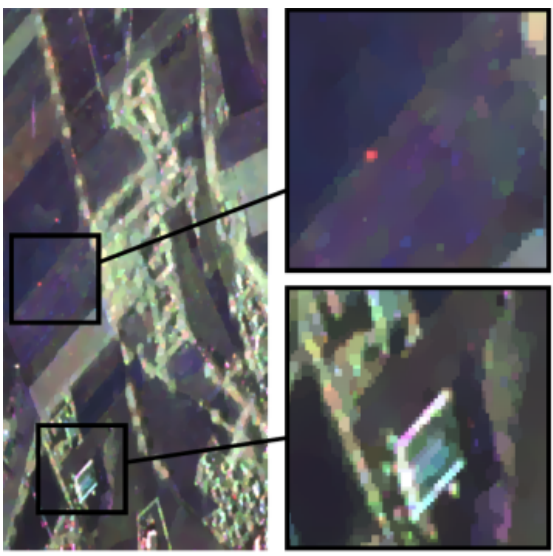

(f) Beltrami

Fig. 13. Results from filtering 3-look L-band E-SAR PolSAR image from Alling, Germany (DLR-HR) [shown in (a)], the images in (b)-(f) show the results using different speckle filtering approaches. 
presents a high-degree of noise correlation. Since no subsampling is performed during the pre-processing step, the Beltrami framework effectively use all the available information. This pre-processing step, however, could introduce unwanted artifacts in the final image. Further analysis to improve the pre-processing step of the presented framework to avoid the introduction of artifacts is planned. The addition of a nonlocal approach instead of a fixed local window to obtain the weighted averages [30], [31] or the utilization of the scattering properties of the data [32] could also alleviate some of the deficiencies of the proposed framework, such as the loss of textural information which could result in an erroneous estimation for certain applications.

A thorough quantitative analysis of the proposed method and other competing state of the art methods was presented. Three different metrics were used to analyze the performance of the Beltrami filtering approach, two of which were proposed as a modification of the log-Euclidean distance and the other is the well known equivalent number of looks. Real word data and synthetic data sets were utilized to assess the performance of the presented filters. The proposed framework presents good results with regards to the metrics utilized, specially showing a high number of looks due to the iterative nature of the filter. The preservation of the eigenvalues, the polarimetric parameters $H / \bar{\alpha} / A$ and the polarimetric complex correlations is also achieved. In this work, we considered both the classical fully-developed case with target vectors following a Gaussian distribution for the first synthetic case, as well as the effects of spatially correlated data in the case of the second synthetic experiment.

Although the Beltrami framework is able to handle $N$ dimensional SAR data (e.g. single intensity SAR data, PolInSAR, TomoSAR, etc...), future work should focus on the analysis and advantages of speckle filtering using the Beltrami approach for interferometric and tomographic applications [31], [33]. However, preliminary results show a good reconstruction of the interferometric coherence in the case of PolInSAR data as well as promising results in the reconstruction of tomograms in the case of TomoSAR, where the despeckling framework was utilized instead of the Boxcar filter as a part of TomoSAR beamforming methods. The effects of noise correlation could be reduced with the addition of a more refined approach in the pre-processing stage of the proposed framework, leading to a better preservation of details in the case of very-high resolution single-look data.

\section{ACKNOWLEDGMENT}

The authors would like to thank the late Yuriy Shkvarko who provided invaluable advice during the development of this work. The authors would also like to thank the Microwave and Radar Institute of the German Aerospace Center (DLR-HR), for providing the Kaufbeuren F-SAR dataset (freely available upon request for research purposes) and the anonymous reviewers for improving the quality of this work.

\section{REFERENCES}

[1] J. Lee and E. Pottier, Polarimetric Radar Imaging: From Basics to Applications, ser. Optical Science and Engineering. CRC Press, 2009.
[Online]. Available: https://books.google.de/books?id=1nAvp2HW $\backslash$ _ gwC

[2] N. D. Mascarenhas, "An overview of speckle noise filtering in SAR images," in Image Processing Techniques, First Latino-American Seminar on Radar Remote Sensing, vol. 407, 1997, p. 71.

[3] F. Argenti, A. Lapini, T. Bianchi, and L. Alparone, "A tutorial on speckle reduction in synthetic aperture radar images," IEEE Geoscience and remote sensing magazine, vol. 1, no. 3, pp. 6-35, 2013.

[4] C. Lopez-Martinez and E. Pottier, "On the extension of multidimensional speckle noise model from single-look to multilook SAR imagery," IEEE Transactions on Geoscience and Remote Sensing, vol. 45, no. 2, pp. 305-320, feb 2007.

[5] J. S. Lee, M. R. Grunes, and G. De Grandi, "Polarimetric SAR speckle filtering and its implication for classification," IEEE Transactions on Geoscience and remote sensing, vol. 37, no. 5, pp. 2363-2373, 1999.

[6] J.-S. Lee, J.-S. Lee, J.-H. Wen, T. L. Ainsworth, K.-S. Chen, and A. J. Chen, "Improved sigma filter for speckle filtering of SAR imagery," IEEE Transactions on Geoscience and Remote Sensing, vol. 47, no. 1, pp. 202-213, Jan 2009.

[7] J. Lee, T. L. Ainsworth, Y. Wang, and K. Chen, "Polarimetric SAR speckle filtering and the extended sigma filter," IEEE Transactions on Geoscience and Remote Sensing, vol. 53, no. 3, pp. 1150-1160, March 2015.

[8] G. Vasile, E. Trouve, J.-S. Lee, and V. Buzuloiu, "Intensity-driven adaptive-neighborhood technique for polarimetric and interferometric SAR parameters estimation," IEEE Transactions on Geoscience and Remote Sensing, vol. 44, no. 6, pp. 1609-1621, June 2006.

[9] O. D'Hondt, S. Guillaso, and O. Hellwich, "Iterative bilateral filtering of polarimetric SAR data," IEEE Journal of Selected Topics in Applied Earth Observations and Remote Sensing, vol. 6, no. 3, pp. 1628-1639, June 2013.

[10] C. Tomasi and R. Manduchi, "Bilateral filtering for gray and color images," in Sixth International Conference on Computer Vision (IEEE Cat. No.98CH36271), Jan 1998, pp. 839-846.

[11] A. Alonso-Gonzalez, C. Lopez-Martinez, and P. Salembier, "Filtering and segmentation of polarimetric SAR data based on binary partition trees," IEEE Transactions on Geoscience and Remote Sensing, vol. 50, no. 2, pp. 593-605, Feb 2012.

[12] F. Lang, J. Yang, D. Li, L. Shi, and J. Wei, "Mean-shift-based speckle filtering of polarimetric SAR data," IEEE Transactions on Geoscience and Remote Sensing, vol. 52, no. 7, pp. 4440-4454, July 2014.

[13] B. Pang, S. Xing, Y. Li, and X. Wang, "Novel polarimetric SAR speckle filtering algorithm based on mean shift," Journal of Systems Engineering and Electronics, vol. 24, no. 2, pp. 222-223, April 2013.

[14] X. Ma, P. Wu, Y. Wu, and H. Shen, "A review on recent developments in fully polarimetric SAR image despeckling," IEEE Journal of Selected Topics in Applied Earth Observations and Remote Sensing, vol. 11, no. 3, pp. 743-758, March 2018.

[15] A. Buades, B. Coll, and J.-M. Morel, "A non-local algorithm for image denoising," in 2005 IEEE Computer Society Conference on Computer Vision and Pattern Recognition (CVPR'05), vol. 2, June 2005, pp. 60-65 vol. 2.

[16] C.-A. Deledalle, L. Denis, F. Tupin, A. Reigber, and M. Jager, "NL-SAR: A unified nonlocal framework for resolution-preserving (Pol)(In)SAR denoising," IEEE Transactions on Geoscience and Remote Sensing, vol. 53, no. 4, pp. 2021-2038, apr 2015.

[17] C. Deledalle, L. Denis, S. Tabti, and F. Tupin, "MuLoG, or how to apply gaussian denoisers to multi-channel SAR speckle reduction?" IEEE Transactions on Image Processing, vol. 26, no. 9, pp. 4389-4403, Sept 2017.

[18] R. Kimmel, R. Malladi, and N. Sochen, Image processing via the Beltrami operator. Berlin, Heidelberg: Springer Berlin Heidelberg, 1997, pp. 574-581. [Online]. Available: https://doi.org/10.1007/3-540-63930-6_169

[19] N. Sochen, R. Kimmel, and R. Malladi, "A general framework for low level vision," IEEE Transactions on Image Processing, vol. 7, no. 3, pp. 310-318, Mar 1998.

[20] A. Spira, R. Kimmel, and N. Sochen, "A short- time Beltrami kernel for smoothing images and manifolds," IEEE Transactions on Image Processing, vol. 16, no. 6, pp. 1628-1636, June 2007.

[21] A. Reigber. (2018) PyRAT (Python Radar Analysis Tools). [Online]. Available: https://github.com/birgander2/PyRAT

[22] N. R. Goodman, "Statistical analysis based on a certain multivariate complex Gaussian distribution (an introduction)," The Annals of Mathematical Statistics, vol. 34, no. 1, pp. 152-177, mar 1963. 
[23] C. Oliver and S. Quegan, Understanding Synthetic Aperture Radar Images, ser. SciTech radar and defense series. SciTech Publ., 2004. [Online]. Available: https://books.google.de/books?id=IeGKe40S77AC

[24] C. Lopez-Martinez, "Multidimensional speckle noise, modelling and filtering related to SAR data," Ph.D. dissertation, Koln, 2004.

[25] C. Lopez-Martinez and X. Fabregas, "Polarimetric SAR speckle noise model," IEEE Transactions on Geoscience and Remote Sensing, vol. 41, no. 10, pp. 2232-2242, Oct 2003.

[26] X. Pennec, P. Fillard, and N. Ayache, "A Riemannian framework for tensor computing," International Journal of Computer Vision, vol. 66, no. 1, pp. 41-66, 2006

[27] S. Kullback and R. A. Leibler, "On information and sufficiency," The annals of mathematical statistics, vol. 22, no. 1, pp. 79-86, 1951.

[28] V. Arsigny, P. Fillard, X. Pennec, and N. Ayache, "Log-euclidean metrics for fast and simple calculus on diffusion tensors," Magnetic resonance in medicine, vol. 56, no. 2, pp. 411-421, 2006.

[29] H. Q. Minh and V. Murino, Covariances in Computer Vision and Machine Learning. Morgan \& Claypool Publishers, 2017.

[30] O. D'Hondt, C. Lpez-Martnez, S. Guillaso, and O. Hellwich, "Impact of non-local filtering on 3D reconstruction from tomographic SAR data," in 2017 IEEE International Geoscience and Remote Sensing Symposium (IGARSS), July 2017, pp. 2476-2479.

[31] O. DHondt, C. Lpez-Martnez, S. Guillaso, and O. Hellwich, "Nonlocal filtering applied to 3-D reconstruction of tomographic SAR data," IEEE Transactions on Geoscience and Remote Sensing, vol. 56, no. 1, pp. 272-285, Jan 2018.

[32] G. D. Martino, A. D. Simone, A. Iodice, and D. Riccio, "Scatteringbased nonlocal means SAR despeckling," IEEE Transactions on Geoscience and Remote Sensing, vol. 54, no. 6, pp. 3574-3588, June 2016.

[33] F. Sica, D. Reale, G. Poggi, L. Verdoliva, and G. Fornaro, "Nonlocal adaptive multilooking in SAR multipass differential interferometry," IEEE Journal of Selected Topics in Applied Earth Observations and Remote Sensing, vol. 8, no. 4, pp. 1727-1742, April 2015.

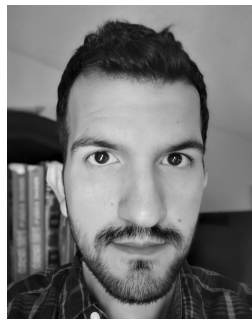

Joel Amao-Oliva received the Dipl.Eng. degree in electronics and communications from the University of Guadalajara, Guadalajara, Mexico, in 2012, the M.Sc. degree in electrical engineering from the CINVESTAV-IPN (Superior Education and Research Center of the National Polytechnic Institute of Mexico), Guadalajara, in 2014, and the Ph.D. degree in electrical engineering from CINVESTAV-IPN in 2018. His research interests include digital signal and image processing for remote sensing applications, SAR polarimetry and SAR interferometry.

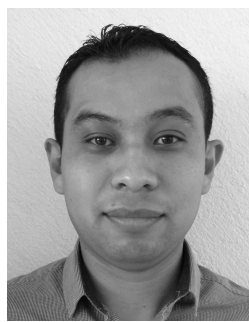

Israel Yanez-Vargas received the degree of Doctor in the Sciences of Electrical Engineering from the Telecommunication Division at the Centro de Investigación y de Estudios Avanzados del Instituto Politécnico Nacional (CINVESTAV-IPN, 2016); the degree of Master of Engineering, Universidad de Guanajuato (UG, 2011); and the degree of Electrical and Electronic Engenineering, Universidad de Guanajuato (UG, 2009). From 2010 to 2011, he was an assistance professor at the Electronic Department at the Universidad de Guanajuato. Presently, he is a full professor in the Universidad Politécnica de Juventino Rosas, Mexico, at the telecommunications and networks engineering department. His research interest are in applications of signal processing in remote sensing, imaging radars, communications, artificial intelligence and medical image processing.

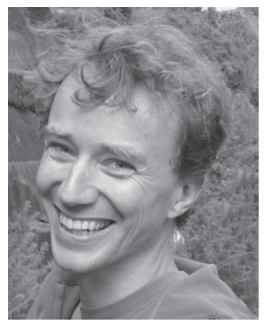

Andreas Reigber (M'02-SM'10-F'16) received the Diploma degree in physics from the University of Constance, Konstanz, Germany, in 1997; the Ph.D. degree from the University of Stuttgart, Stuttgart, Germany, in 2001; and the Habilitation from the Berlin University of Technology, Berlin, Germany, in 2008. He is currently the head of the SAR Technology Department, Microwave and Radar Institute, German Aerospace Center (DLR), Wessling, Germany, and a Professor of remote sensing and digital image processing with Technische Universität Berlin, Berlin, Germany. His current research interests include the various aspects of multimodal and multichannel SAR, and filtering and classification aspects of high-resolution SAR data. Prof. Reigber has received several prize paper awards, among them the IEEE TGRS Transactions Prize Paper Award in 2001 for a work on polarimetric SAR tomography and the IEEE TGRS Letters Prize Paper Award in 2006 for a work on multipass SAR processing.

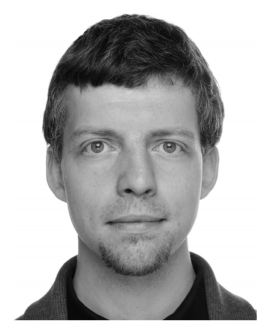

Marc Jäger received the B.A. (Hons.) degree in computer science from the University of Cambridge, Cambridge, U.K., in 2001, and the M.Sc. (with distinction) degree in digital signal and image processing from De Montfort University, Leicester, U.K., in 2003. He is currently with the German Aerospace Center (DLR), Wessling, Germany, focusing on the polarimetric and interferometric calibration and processing of airborne synthetic aperture radar (SAR) data. His research interests include the automatic extraction of semantic information from polarimetric Telecommunication Research Prize in 1993 from AHCIET Association and was recipient of the 1995 best Paper Award from AHCIET Review, Spain. Since 1996, he has been an associate professor at CINVESTAV-IPN, Guadalajara, Mexico. His research interests include hardware and software deand polarimetric interferometric SAR data using machine learning techniques. 